\title{
Loosely bound hyperons in the SU(3) Skyrme model
}

\author{
B. Schwesinger ${ }^{1,2 *}$, F.G. Scholtz ${ }^{1 \dagger}$ and H.B. Geyer ${ }^{1 \ddagger}$ \\ ${ }^{1}$ Institute of Theoretical Physics, University of Stellenbosch, Stellenbosch 7600, South Africa \\ ${ }^{2}$ Siegen University, Fachbereich Physik, 57068 Siegen, Germany
}

\begin{abstract}
Hyperon pairs bound in deuteron like states are obtained within the SU(3) Skyrme model in agreement with general expectations from boson exchange models. The central binding from the flavor symmetry breaking terms increases with the strangeness contents of the interacting baryons whereas the kinetic non-linear $\sigma$-model term fixes the spin and isospin of the bound pair. We give a complete account of the interactions of octet baryons within the product approximation to baryon number $B=2$ configurations.
\end{abstract}

PACS numbers 13.75.Ev, 13.75.Cs, 12.39.Dc

\footnotetext{
*Electronic address: schwesinger@hrz.uni-siegen.d400.de

†Electronic address: fgs@sunvax.sun.ac.za

‡Electronic address: hbg@sunvax.sun.ac.za
} 


\section{INTRODUCTION}

It seems to be generally recognized that the relative scarcity of experimental data on hyperon-nucleon and especially hyperon-hyperon interactions calls for major theoretical input to arrive at a satisfactory description of the data [1]. From the boson exchange point of view such a description is typically approached by "upgrading" a reliable description of the $N N$-interaction with $\mathrm{SU}(3)$ flavor symmetry, assuming of course that the formulation of the $N N$-interaction admits such a generalization. An example of this approach can be found in the construction of the Nijmegen potentials (see Ref. [1] and references therein). The one-boson exchange potentials obtained in this way generally predict the existence of loosely bound hyperons, while ruling out deeply bound hyperons.

An alternative approach which is well tailored to address the construction of hyperonnucleon and hyperon-hyperon potentials is one based on the Skyrme model [2]. It has already been successfully implemented in the construction of the nucleon-nucleon potential, as reviewed recently by Walhout and Wambach [0]. In the Skyrme model deeply bound hyperons also seem to be excluded once Casimir energies are properly taken into account [6], but deuteron-like states, for which a Skyrmion product ansatz description seems to suffice, remain possible. An analysis of such configurations to determine hyperon-nucleon and hyperon-hyperon potentials have in fact recently been carried out by Kälbermann and Eisenberg [7],8] where much of the focus is on the central interactions. No conclusive statements about the existence or otherwise of bound states seem to have emerged from these two studies.

Before we indicate below that the SU(3) Skyrme model construction of hyperon-nucleon and hyperon-hyperon potentials can indeed lead to qualitative and quantitative results, it is perhaps worthwhile to point out that such an approach has the further merit that it ties together the mesonic sector $(B=0)$, the baryonic sector $(B=1)$ and the $B=2$

sector in the single comprehensive framework of a dynamically closed model with only a few (typically 5) parameters. Furthermore the SU(3) flavor symmetry breaking part is fixed 
by meson masses and weak decay constants, while in turn it then reproduces the hyperon spectrum in the $B=1$ sector and will also be responsible for two hyperons forming loosely bound deuteron-like states.

We now turn to some of the more salient aspects of our analysis. It is rather satisfactory to find that, apart from the known problems associated with the central attraction in the Skyrme model (see Sec. IV for further discussion of this point), many of the more robust predictions from the one-boson exchange approach to the problem also emerge from the Skyrme model analysis. In this regard we mention that we extract unambiguously an attractive $\Lambda \Lambda$ interaction in the ${ }^{1} S_{0}$ state, a result also required from a number of complementary analyses [9]. Secondly we find e.g. an $N \Sigma$ potential with relative components of the various spin and isospin interactions which are comparable with those found in the Nijmegen potentials [10,11]. As another specific example, the spin dependence of the $N \Lambda$ potential we obtain is the same as that favoured from phenomenological and potential model estimates in Ref. [12]. Furthermore it is possible to predict from our analysis the spin and isospin of bound deuteron type hyperon states. We also extract a rather prominent correlation of an increased binding of baryons with growing strangeness.

A short outline of the presentation is as follows. In Secs. [I] and III we introduce the $\mathrm{SU}(3)$ lagrangian, recall aspects of the framework in which it is typically employed and discuss our strategy of identifying a set of independent relative orientations of two skyrmions which eventually facilitates the identification of the various interaction potentials. This strategy, which generalises the known approach for the $N N$-potential [5, 13], seems to offer advantages over the approach of Refs. [7, [8] where a Monte Carlo calculation is used to evaluate matrix elements [14]. (See also Sec. [V.) Detail of this rather technical part is collected in Appendix A. In Sec. $\mathbb{\nabla}$ we consider the question of missing intermediate range attraction in the Skyrme model and possible resolutions, argue to which extent various aspects of our present analysis of hyperon-hyperon interactions would retain their validity should this be resolved satisfactorily, and then discuss general and specific results for the various interactions. Tables for the various interaction potentials as function of the separation $R$ 
are collected in Appendix B.

\section{LAGRANGIAN AND $B=1$ SECTOR}

The lagrangian we use is the standard SU(3) Skyrme-model lagrangian given by [15, 16]

$$
\mathcal{L}=\mathcal{L}^{(\mathrm{SYM})}+\mathcal{L}^{(\mathrm{FSB})}
$$

In terms of the chiral field $U$ and with the standard notation for the left current

$$
L_{\mu}=U^{\dagger} \partial_{\mu} U=i \lambda_{a} L_{\mu}^{a}
$$

and the topological current

$$
B^{\mu}=\frac{\varepsilon^{\mu \nu \alpha \beta}}{24 \pi^{2}} \operatorname{tr} L_{\nu} L_{\alpha} L_{\beta}
$$

the flavor symmetric and flavor symmetry breaking parts are given by

$$
\begin{aligned}
\mathcal{L}^{(\mathrm{SYM})}(U)= & -\frac{f_{\pi}^{2}}{4} \operatorname{tr} L_{\mu} L^{\mu}+\frac{1-\chi}{32 e^{2}} \operatorname{tr}\left[L_{\mu}, L_{\nu}\right]\left[L^{\mu}, L^{\nu}\right] \\
& +\frac{\chi}{16 e^{2}}\left\{\left(\operatorname{tr} L_{\mu} L^{\mu}\right)^{2}-\operatorname{tr} L_{\mu} L_{\nu} \cdot \operatorname{tr} L^{\mu} L^{\nu}\right\} \\
& -\frac{\varepsilon_{6}^{2}}{2} B_{\mu} B^{\mu}+\frac{m_{\pi}^{2} f_{\pi}^{2}}{4} \operatorname{tr}\left(U+U^{\dagger}-2\right) \\
\equiv & \mathcal{L}^{(2)}+\mathcal{L}^{(4 \mathrm{~A})}+\mathcal{L}^{(\widetilde{4 \mathrm{~A}})}+\mathcal{L}^{(6)}+\mathcal{L}^{(\mathrm{CSB})}
\end{aligned}
$$

and

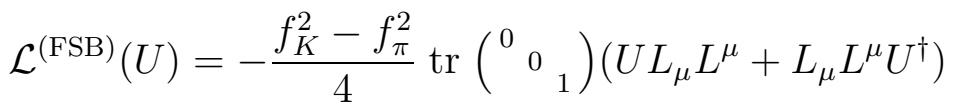

$$
\begin{aligned}
& +\frac{f_{K}^{2} m_{K}^{2}-f_{\pi}^{2} m_{\pi}^{2}}{2} \operatorname{tr}\left(\begin{array}{lll}
0 & & \\
& 0 & \\
& & 1
\end{array}\right)\left(U+U^{\dagger}-2\right),
\end{aligned}
$$

where we have provided for the possibility of different stabilizing terms at most quadratic in the time derivatives. Among the stabilizers of fourth order the extension to $\mathrm{SU}(3)$ allows for an alternate term $\mathcal{L}^{(\widetilde{4 A})}\left[17\right.$ which reduces to the Skyrme term $\mathcal{L}^{(4 \mathrm{~A})}$ in the $\mathrm{SU}(2)$-limit. It's effects in the $B=1$ sector [16] have been small, whereas here they are shown to be large if $\chi$ is chosen accordingly. 
The lagrangian (1) succesfully describes the baryon splittings in the $B=1$ sector when empirical meson masses and decay constants are used. Fig. 11 shows the baryon spectrum calculated by treating the flavor symmetry breaking in two different ways: for rigidly rotated solitons (RRA) [18, 19, 16] the chiral angle minimizing the soliton in the isospin subgroup is rotated without changes into flavor directions of decreasing hypercharge. In case of the slow rotator approach (SRA) 20,21] the soliton is allowed to change its shape according to the flavor symmetry breaking forces excerted at a given strangeness angle. These forces mainly reduce the one pion tail of the $\mathrm{SU}(2)$ soliton to a more rapid decay corresponding to the higher meson masses present in the cloud.

\section{PRODUCT ANSATZ, ENERGY FUNCTIONAL AND ASYMPTOTIC INTERACTION IN THE $B=2$ SECTOR}

In order to obtain a hyperon-hyperon potential (generally baryon-baryon potential) we follow the standard Skyrme model procedure, namely evaluate the energy of a $B=2$ configuration and subtract the energies of the two $B=1$ configurations which constitute that $B=2$ configuration. As the extremely short distance behaviour of the potentials to be calculated is not of immediate concern, we content ourselves with the following hedgehog product ansatz for the $B=2$ configuration

$$
U(\boldsymbol{r} ; A, C)=A U_{\mathrm{H}}\left(x, y, z-\frac{R}{2}\right) C U_{\mathrm{H}}\left(x, y, z+\frac{R}{2}\right) C^{\dagger} A^{\dagger}
$$

where $U_{\mathrm{H}}(\boldsymbol{r})=e^{i \boldsymbol{\tau} \cdot \hat{r} \chi(r)}$. $A$ and $C=A^{\dagger} B$ are constant flavor rotations, $C$ denoting the relative orientation of the two $\mathrm{SU}(3)$ skyrmions in flavor space. As a matter of convenience we chose the two skyrmions to be separated by a distance $R$ along the $z$-axis. The profile function for the hedgehog is obtained by a numerical minimization of the energy functional in the $B=1$ sector. Using the product ansatz we can calculate the energy of the $B=2$ configuration for any given orientations $A, C$ and separation $R$ by performing a numerical integration over the spatial coordinates of the hedgehog configuration. 
One notes from translation and flavor symmetry that the energy functional resulting from $\mathcal{L}^{(\mathrm{SYM})}$ depends only on the relative separation $R$ and orientation $C$ of the two skyrmions. The general structure of the energy functional is therefore established in the form

$$
\begin{aligned}
V^{(\mathrm{SYM})}(R ; A, C)= & U^{(0)}(R)+U_{S}^{(1)}(R) D_{i i}(C)+\left(3 D_{33}(C)-D_{i i}(C)\right) U_{T}^{(1)}(R) \\
+ & \sum_{(I)=(4 A),(\widetilde{4 A}),(6)}\left\{U_{S}^{(2, I)}(R) Q^{(I)} \frac{1}{9} D_{i j}(C) D_{i j}(C)\right. \\
& +U_{S^{\prime}}^{(2, I)}(R) Q^{(I)} \frac{1}{4}\left(D_{i i}(C) D_{j j}(C)-D_{i j}(C) D_{j i}(C)\right) \\
& +U_{T}^{(2, I)}(R) Q^{(I)} \frac{1}{4}\left(6 D_{11}(C) D_{22}(C)-6 D_{12}(C) D_{21}(C)\right. \\
& \left.\left.\quad-D_{i i}(C) D_{j j}(C)+D_{i j}(C) D_{j i}(C)\right)\right\} \\
& +U_{S}^{(3)}(R) D_{88}(C)
\end{aligned}
$$

Here we have used the convention $(i, j) \in\{1,2,3\}$ and the notation

$$
Q^{(I)}=\left\{\begin{array}{cl}
3-\frac{1}{2} L_{a}^{2} & ,(I)=(4 A) \\
1, & (I)=(\widetilde{4 A}),(6)
\end{array}\right.
$$

where $L_{a}^{2}$ is the Casimir operator of $\mathrm{SU}(3)$ and $D_{a b}$ the $\mathrm{SU}(3) D$-functions in the adjoint representation. The occurence of the Casimir operator of $\mathrm{SU}(3)$ is due to a manipulation which allows us to keep the indices of the $D$-functions in the $\mathrm{SU}(2)$-subgroup: the energy density from the Skyrme term $\mathcal{L}^{(4 \mathrm{~A})}$ has contributions proportional to

$$
\begin{aligned}
f_{a b c} D_{c c^{\prime}} f_{a d e} D_{e e^{\prime}} & =-\left(L_{a} D_{b c^{\prime}}\right)\left(L_{a} D_{d e^{\prime}}\right) \\
& =-\frac{1}{2} L_{a}^{2}\left(D_{b c^{\prime}} D_{d e^{\prime}}\right)+\frac{1}{2}\left(L_{a}^{2} D_{b c^{\prime}}\right) D_{d e^{\prime}}+\frac{1}{2} D_{b c^{\prime}}\left(L_{a}^{2} D_{d e^{\prime}}\right)
\end{aligned}
$$

where $L_{a}$ are the left Euler angular momentum operators. The explicit expressions stemming from the action of the Casimir operators are given in Appendix A.

In Eq. (7) we do not show those terms which contain combinations of $D$-functions which are of second rank in the spin in the energy functional, since their matrix elements between the spin one-half baryon states vanish. In principle they do, however, occur and due care has to be exercised, when the different components of the interaction are evaluated. For 
completeness we list these combinations ordered according to the spin-tensorial decomposition of the left and right indices of the two $D$-functions which refer to the Euler angles in $A$ and $B$ respectively.

$$
\begin{array}{ll}
{[0 \times 2]_{2}:} & D_{i 3} D_{i 3}-\frac{1}{3} D_{i j} D_{i j}, \\
{[2 \times 0]_{2}:} & D_{3 i} D_{3 i}-\frac{1}{3} D_{i j} D_{i j}, \\
{[2 \times 2]_{0}:} & \frac{1}{2} D_{i i} D_{j j}+\frac{1}{2} D_{i j} D_{j i}-\frac{1}{3} D_{i j} D_{i j}, \\
{[2 \times 2]_{2}:} & \frac{1}{2} D_{i i} D_{33}+\frac{1}{2} D_{i 3} D_{3 i}-\frac{1}{3} D_{i 3} D_{i 3}-\frac{1}{3} D_{3 i} D_{3 i}+\frac{1}{9} D_{i j} D_{i j} \\
{[2 \times 2]_{4}:} & D_{33} D_{33}-\frac{1}{3} D_{i 3} D_{i 3}-\frac{1}{3} D_{3 i} D_{3 i}+\frac{1}{9} D_{i j} D_{i j} .
\end{array}
$$

The seven different components $U^{(0)}(R), U_{S}^{(1)}(R) \ldots U_{T}^{(2)}(R)$ of the interaction appearing in $V^{(\mathrm{SYM})}(R ; A, C)$ can now be calculated. We note that if we evaluate the energy of the $B=2$ hedgehog-hedgehog configuration for twelve orientations, $C$, such that the seven combinations of $D$-functions appearing in $V^{(\mathrm{SYM})}(R ; A, C)$ and the five combinations of Eq. (10) are linearly independent, we obtain a system of linear equations from which the interaction components can be solved by inversion of the coefficient matrix. The set of orientations

$$
\begin{aligned}
& 1, \quad e^{i \frac{\pi}{2} \lambda_{2}}, \quad e^{i \frac{\pi}{2} \lambda_{3}}, \quad e^{i \frac{\pi}{2} \lambda_{4}} e^{i \frac{\pi}{4} \lambda_{2}}, \quad e^{i \frac{2 \pi}{3} \lambda_{2}} e^{i \frac{\pi}{2} \lambda_{4}}, \quad e^{i \frac{\pi}{4} \lambda_{4}} e^{i \frac{\pi}{2} \lambda_{2}} e^{-i \frac{\pi}{4} \lambda_{4}}, \\
& e^{i \frac{\pi}{4} \lambda_{4}} e^{i \frac{\pi}{2} \lambda_{3}} e^{-i \frac{\pi}{4} \lambda_{4}}, \quad e^{i \frac{\pi}{4} \lambda_{1}} e^{i \frac{\pi}{4} \lambda_{4}} e^{i \frac{\pi}{4} \lambda_{2}}, \quad e^{i \frac{\pi}{6} \lambda_{4}} e^{i \frac{\pi}{5} \lambda_{6}} e^{i \frac{\pi}{5} \lambda_{4}}, \quad e^{i \frac{\pi}{6} \lambda_{1}} e^{i \frac{\pi}{5} \lambda_{6}} e^{i \frac{\pi}{5} \lambda_{4}}, \\
& e^{i \frac{\pi}{6} \lambda_{2}} e^{i \frac{\pi}{5} \lambda_{6}} e^{i \frac{\pi}{5} \lambda_{4}}, \quad e^{i \frac{\pi}{6} \lambda_{3}} e^{i \frac{\pi}{5} \lambda_{6}} e^{i \frac{\pi}{3} \lambda_{4}} .
\end{aligned}
$$

can be checked to give the required linear independence and are subsequently used to determine the interaction components as already outlined.

Translation invariance implies that for the flavor symmetry breaking part $\mathcal{L}^{(\mathrm{FSB})}$, the energy functional depends on $R$ alone, but since flavor symmetry is broken, it depends on the orientations $A$ and $B$ of the individual skyrmions in flavor space. Neglecting the numerically small and cumbersome terms proportional to $f_{K}^{2}-f_{\pi}^{2}$, a simple calculation shows the energy functional to be 


$$
\begin{aligned}
V^{(\mathrm{FSB})}(R ; A, B)=U_{0}^{(\mathrm{FSB})}(R) & {\left[\left(1-D_{88}(A)\right)\left(1-D_{88}(B)\right)+\frac{3}{4} D_{k 8}(A) D_{k 8}(B)\right] } \\
+ & U_{1}^{(\mathrm{FSB})}(R)\left[D_{8 i}(A) D_{8 i}(B)+\frac{3}{4} D_{k i}(A) D_{k i}(B)\right] \\
+ & U_{2}^{(\mathrm{FSB})}(R)\left[\left(3 D_{83}(A) D_{83}(B)-D_{8 i}(A) D_{8 i}(B)\right)\right. \\
& \left.+\frac{3}{4}\left(3 D_{k 3}(A) D_{k 3}(B)-D_{k i}(A) D_{k i}(B)\right)\right],
\end{aligned}
$$

where $k \in\{4,5,6,7\}, i \in\{1,2,3\}$. The radial functions in the flavor symmetry breaking part of the energy functional, $V^{(\mathrm{FSB})}(R ; A, B)$, are given in terms of the integrals

$$
\begin{aligned}
U_{0}^{(\mathrm{FSB})}(R)= & \frac{4}{9}\left(f_{K}^{2} m_{K}^{2}-f_{\pi}^{2} m_{\pi}^{2}\right) \int-\left(1-c_{1}\right)\left(1-c_{2}\right) d^{3} r \\
U_{1}^{(\mathrm{FSB})}(R)= & \frac{4}{9}\left(f_{K}^{2} m_{K}^{2}-f_{\pi}^{2} m_{\pi}^{2}\right) \int \hat{r}(1) \cdot \hat{r}(2) s_{1} s_{2} d^{3} r \\
U_{2}^{(\mathrm{FSB})}(R)= & \frac{4}{9}\left(f_{K}^{2} m_{K}^{2}-f_{\pi}^{2} m_{\pi}^{2}\right) \int \frac{1}{2}\left(3 \hat{r}_{3}(1) \hat{r}_{3}(2)-\hat{r}(1) \cdot \hat{r}(2)\right) s_{1} s_{2} d^{3} r, \\
& \boldsymbol{r}(i)=\boldsymbol{r} \pm \frac{R}{2} \boldsymbol{e}_{3}, \quad c_{i}=\cos \chi(r(i)), \quad s_{i}=\sin \chi(r(i)) \quad i=1,2 .
\end{aligned}
$$

These integrals are evaluated numerically.

Finally, knowing the interaction components, we have to evaluate the matrix elements of the energy functional between the spin one-half baryon wavefunctions diagonalizing the rotational hamiltonian with the symmetry breaking term, see 18, 19, 16] and Eq. (A1) to extract the potentials in the various interaction channels. We have to distinguish direct and exchange matrix elements, see Fig. 2. The single particle degrees of freedom of the interacting baryons are given by the collective rotations $A$ and $B$ respectively, which appear in all the $D$-functions of the potential energy. In such an interaction term $D(A)$, say, can transfer strangeness $0 \leq|S| \leq 1$ to the incoming baryon state depending on the Euler angles $A$. For the direct terms the outgoing baryon also carrying the Euler angles $A$ has the same hypercharge as the incoming one, whereas the exchange terms allow for different strangeness of the outgoing baryon. Since total strangeness is, of course, conserved, the baryon described by the Euler angles $B$ must compensate for the hypercharge difference. Speaking in terms of a one boson exchange description, the latter processes thus contain the kaon exchange terms. 
The details of the calculation of the matrix elements and the various interaction potentials are collected in Appendix A. We must emphasize, however, that generally, we will only use wave functions diagonalizing the rigid rotator hamiltonian, because the use of a chiral angle which changes in response to the flavor symmetry breaking forces [20,21] is technically too complicated, here. For testing purposes we will also use baryon wavefunctions which are $\mathrm{SU}(3)$ symmetric as well as those for the strong symmetry breaking limit. Unfortunately, the restriction to the rigidly rotating soliton directly leads to the fact that the asymptotic forces will always have the one-pion exchange tail, even for kaon exchange terms. This statement can be verified by examination of the asymptotic interaction, which is related to the lower order terms in the lagrangian after insertion of the equations of motion for the individual solitons. Within $\mathrm{SU}(2)$ this has been shown by Yabu and Ando [22], and the extension to $\mathrm{SU}(3)$ is straight-forward:

$$
\begin{aligned}
& V(R ; A, B) \stackrel{R \rightarrow \infty}{\longrightarrow}
\end{aligned}
$$

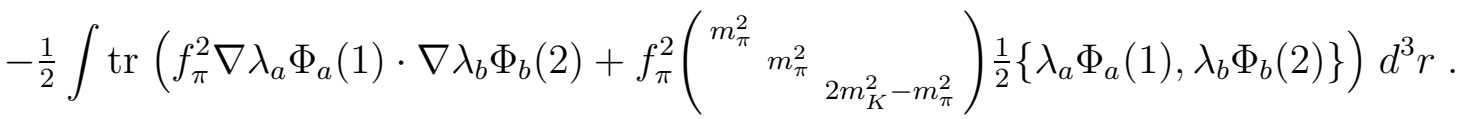

The eight asymptotic pseudoscalar soliton fields $\Phi_{a}$ solving the Euler Lagrange equations should have the formf

$$
\Phi_{a}=D_{a b} \hat{r}_{b} \begin{cases}\chi_{\pi} & a \in\{1,2,3\} \\ \chi_{K} & a \in\{4,5,6,7\} \\ \chi_{\eta} & a=8\end{cases}
$$

with

$$
\hat{r} \chi_{C}=-\nabla A_{C} \frac{\mathrm{e}^{-m_{C} r}}{r}
$$

leading to

\footnotetext{
${ }^{1}$ We thank A. Hayashi for supplying us the asymptotic solution of the Euler Lagrange equations when flavor symmetry is broken.
} 


$$
\begin{aligned}
& V(R ; A, B) \stackrel{R \rightarrow \infty}{\longrightarrow} \\
& 4 \pi f_{\pi}^{2} \nabla_{i} \nabla_{j}\left\{\begin{array}{l}
D_{k i}(A) D_{k j}(B) A_{K}^{2} \frac{\mathrm{e}^{-m_{K} R}}{R} \\
\left.\quad+D_{8 i}(A) D_{8 j}(B) A_{\eta}^{2} \frac{\mathrm{e}^{-m_{\eta} R}}{R}+D_{i^{\prime} i}(A) D_{i^{\prime} j}(B) A_{\pi}^{2} \frac{\mathrm{e}^{-m_{\pi} R}}{R}\right\}
\end{array}\right.
\end{aligned}
$$

However, due to the rigid rotator approximation all masses are set to $m_{\pi}$ and the asymptotic constants all equal

$$
A_{\mathrm{SU}(2)}=\frac{g_{\pi N N}\left(-m_{\pi}^{2}\right)}{g_{\pi N N}(0)} \frac{3 g_{A}^{\mathrm{SU}(2)}}{8 \pi f_{\pi}^{2}}
$$

For SU(3)-symmetry the matrix elements of the $D$-functions in Eq. (17) for nucleons are summarized by

$$
D_{i j}(C)=D_{a i}(A) D_{a j}(B) \rightarrow\left(\frac{7}{30}\right)^{2} \sigma_{i}(A) \sigma_{j}(B)\left[\boldsymbol{\tau}(A) \cdot \boldsymbol{\tau}(B)+\frac{3}{49}\right]
$$

and we recover the conventional one pion exchange interaction together with a small term from one $\eta$ exchange since for $\mathrm{SU}(3)$-symmetry $g_{A}^{\mathrm{SU}(3)}=\frac{7}{10} g_{A}^{\mathrm{SU}(2)}$ in the Skyrme model.

Numerically and fortunately, the long range part of the exchange matrix elements will turn out to be small, so the impact of the wrong tail is not important.

\section{RESULTS}

The main emphasis of the present investigation is on the question of whether strangeness is loosely bound. Accessing the problem within the framework of the Skyrme model has some advantageous and some problematic aspects.

The problematic aspects stem from uncertainties in the higher order terms of the lagrangian, inadequacies of the product ansatz and, ultimately, the disturbing feature that an intermediate range attraction in the nucleon-nucleon system does not emerge as simply and naturally as one might have hoped, see e.g. [5, 13, 23, 24] and references therein. The obvious advantages lie in the fact that the lower order terms of the lagrangian are unambiguously fixed in the mesonic winding number zero sector. Now, the same lagrangian governs the 
higher winding number configurations, so that conclusions based solely on the lower order terms, should also be reliable for the case of higher winding numbers. One example for this is the baryon spectrum in $\mathrm{SU}(3)$, where the lower order $\mathrm{SU}(3)$ symmetry breaking in the lagrangian, Eq. (5), is sufficient to describe the mass splittings, as recalled in Sec. II. Another example is given in the case of meson-baryon scattering where the use of mesonic weak decay constants and masses for this winding number one configuration is mandatory 25,26 .

Provided that the problem of the missing intermediate range attraction finds its resolution in higher order terms and/or in improved two-baryon configurations [5] beyond the product ansatz, we can confidently focus on the longer range part of the interaction: it does not involve the higher order terms explicitely and also the product ansatz gradually becomes exact.

The longer range components of the baryon-baryon forces are due to the flavor symmetric and flavor symmetry breaking mass terms $\mathcal{L}^{(\mathrm{CSB})}$ and $\mathcal{L}^{(\mathrm{FSB})}$, and the non-linear $\sigma$-model term $\mathcal{L}^{(2)}$, see Eq. (14), where the latter does not contribute at all to the central interaction. If we accept the line of reasoning above, the central interactions from the flavor and chirally symmetric terms of the lagrangian are viable to modifications, the others not.

In Figs. 3-4 we show the central interactions from the flavor symmetric part of the lagrangian for the $N N_{-}, \Lambda \Lambda$-, $\Sigma \Sigma$ - and $\Xi \Xi$-systems. The general absence of any attraction is as conspicuous as the fact, that the variation with respect to decreasing hypercharge of the system is small. This suggests, that any improvement on the central attraction in the $N N$-system will lead to very similar attractions for the other baryons.

We explore this hypothesis in Figs. 3-4 by varying the higher order stabilizing terms of the lagrangian: Fig. 3 shows the changes when the usual Skyrme term $\mathcal{L}^{(4 \mathrm{~A})}$, i.e. $\chi=0$ in Eq. (4), is replaced by its alternate form $\mathcal{L}^{(\widetilde{4 A})}$, i.e. $\chi=1$ in Eq. (4). This substitution does not lead to any changes within $\mathrm{SU}(2)$, but in $\mathrm{SU}(3)$ the situation changes drastically as may be seen from the figure. Since the $B=1$ solitons in the $\mathrm{SU}(2)$-subgroup are unaffected by variations of $\chi$ it is clear that large negative values of $\chi$ will yield arbitrary attraction 
for $B=2$-configurations in $\mathrm{SU}(3)$. But it seems very likely to us, that the $B=1$ soliton would not be stable towards deformations out of the SU(2)-subgroup then, to which it was constrained by ansatz. We therefore refrain from such manipulations. In any case, from the mesonic sector, $\mathcal{L}^{(\widetilde{4 A})}$ seems to be small [17] since it violates the Zweig rule.

Fig. 4 shows the changes, when the fourth order stabilizer is replaced by a sixth order one and when scalar degrees of freedom are taken into account. For the latter, we choose a form inspired by, but simpler than, a local approximation to the dilatons [27,28,24], namely

$$
\begin{aligned}
\mathcal{L}^{(2)}(U) & \rightarrow \widetilde{\mathcal{L}}^{(2)}(U) \\
& =-\frac{f_{\pi}^{2} m_{\pi}^{2}}{4 \kappa^{2}}\left(1-\exp \left(-\frac{\kappa^{2}}{m_{\pi}^{2}} \operatorname{tr} L_{\mu} L^{\mu}\right)\right),
\end{aligned}
$$

which effectively scales down $f_{\pi}$ in the interior of the soliton. Expanding the exponential to second order gives the non-linear $\sigma$-model and the usual attractive symmetric fourth order term which destabilizes the soliton, but here there are higher order terms present in the exponential to counteract this destabilization now. Within $\mathrm{SU}(2)$ the inclusion of dilatons was shown to lead to an intermediate range central attraction [24] when these fields were made strong enough. In $\mathrm{SU}(3)$, however, strong dilaton fields lead to a problem since not only $f_{\pi}$ is scaled down in the interior but also all mass terms resulting in too small hyperon splittings in the $B=1$ sector $[29$.

As already mentioned, the rationale of all these different higher order terms is not only to investigate further mechanisms for an intermediate range attraction, but also to demonstrate that the central interaction from the flavor symmetric terms is fairly independent of the hypercharge of the two-baryon configuration. Thus, if the $N N$-system acquires it's intermediate range attraction from the flavor symmetric terms, this attraction will also be present for the hyperons. It remains to be said, that the interactions in Figs. 3-4 have all been determined with baryon wavefunctions diagonalizing the rotational motion in the presence of SU(3) symmetry breaking, see Eq. (A1). The distortions in the baryon wavefunctions away from $\mathrm{SU}(3)$, however, do not play a decisive role here, which means, that $\mathrm{SU}(3)$ symmetric wavefunctions or wavefunctions in the strong symmetry breaking limit always lead to very 
small variations of the flavor symmetric central interaction with respect to hypercharge.

Fig. 5 shows the central interactions for identical baryons as they arise from those terms, which explicitly break the flavor symmetry. These contributions arise solely from the Euler angle matrix elements of

$$
U_{0}^{(\mathrm{FSB})}(R)\left(1-D_{88}(A)\right)\left(1-D_{88}(B)\right)
$$

in Eq. (12) and are directly proportional to the product of the strangeness contents of the two interacting baryons, since $\langle Y|\bar{s} s| Y\rangle=\left\langle Y\left|\frac{1}{3}\left(1-D_{88}\right)\right| Y\right\rangle$. From Fig. 5 we see that $U_{0}^{(\mathrm{FSB})}(R)$ is attractive and large, such that binding is enhanced with growing strangeness content. Since this additional binding comes from a term fixed in the mesonic sector, we judge this to be a reliable conclusion, which also summarises one of the main points of the present work.

In Appendix $\mathrm{B}$ we give a complete list of all interaction components for $N N, N \Lambda, N \Sigma$, $N \Xi, \Lambda \Lambda, \Sigma \Sigma$ and $\Xi \Xi$ systems using only the normal Skyrme term, $\mathcal{L}^{(4 \mathrm{~A})}$, as stabilizer adjusting the $N \Delta$-splitting. Again, the longer-range spin-isospin dependence is due to terms fixed in the mesonic sector and thus insensitive to any reasonable modifications of the lagrangian. For the $N N$-case we exploit one of the advantages of our approach to the $\mathrm{SU}(3)$-interactions, namely that we are able to switch continuously from SU(3)-symmetry to the $\mathrm{SU}(2)$-limit by simply changing the baryon wavefunctions, see Eq. (A1). Comparing these cases for the $N N$-interaction, we find that there is a smooth transition with small changes in the longer range parts of the interaction to the well known results in $\mathrm{SU}(2)$. This finding apparently is in contrast to other work published recently on the baryon-baryon interactions in the $\mathrm{SU}(3)$ Skyrme model [7,8] and similar discrepancies persist for other baryon pairs, too, which could be [14] due to difficulties in the Monte Carlo integrations used in ref. [7],8].

From the fact that two nucleons are loosely bound and well separated in an isospin $I=0$, spin $J=1$ state (the deuteron), and that no additional repulsion arises in the longer-range central interactions when hypercharge is decreased, we are able to predict the spin and isospin of bound deuteron-like states of hyperons. To this end we just have to examine the 
sign of the different spin-isospin components of the long-range interaction, which, as already has been stated, is due to well established terms in the lagrangian. Explicitly (see Appendix B) we find that an $N \Lambda$-pair should be bound in a $\left(I=\frac{1}{2}, J=1\right)$-state, an $N \Sigma$-pair in a $\left(I=\frac{1}{2}, J=1\right)$-state, an $N \Xi$-pair in a $(I=0, J=0)$-state, a $\Lambda \Lambda$-pair in a $(I=0, J=0)$ state, a $\Sigma \Sigma$-pair in a $(I=2, J=0)$-state, and a $\Xi \Xi$-pair in a $(I=1, J=0)$-state.

In $\mathrm{SU}(2)$ the most attractive configuration, the torus, can be found numerically by initializing a general minimization algorithm with the most attractive product configuration [30,31,5]. This most attractive product configuration occurs at a relative orientation of

$C=\exp \left(i \frac{\pi}{2} \lambda_{2}\right)$ for the two hedgehogs. With the tools set up for the present investigation we are in a position to determine the most attractive product configuration in $\mathrm{SU}(3)$ by minimizing the flavor symmetric part of the interaction energy, Eq. (7) with respect to the 8 Euler angles in $C$ at all separations. Interestingly, the most attractive orientation is unchanged, i.e. it resides in the $\mathrm{SU}(2)$ subgroup, hinting that the torus will also be the minimum in the $B=2$-sector of $\mathrm{SU}(3)$. In case of flavor symmetry with all meson masses equal to the pion mass the torus is known [6] to be lower in energy than the $\mathrm{SO}(3)$-soliton found by Balachandran et al. [32].

\section{CONCLUSIONS}

In the present work we have explored to which extent it is possible to make reliable statements about whether hyperons form bound deuteron like states. To this end we have used the $\mathrm{SU}(3)$ Skyrme model and compared the $N N$-interaction in the product ansatz to different $N Y$ - and $Y Y$-interactions which emerge from the same ansatz. ( $Y$ represents any octet baryon with non-zero strangeness.) For loosely bound deuteron like configurations the product ansatz is sufficient if the only purpose is the investigation of whether there is more binding in the $N Y$-system relative to the $N N$-case in the longer range part of the interaction.

Such a comparison within the Skyrme model unfortunately also meets two other obstruc- 
tions: the well-known problem of the missing intermediate range central attraction and the incomplete knowledge on the correct combination of higher order stabilizing terms. The latter statement, of course, reflects the belief that the lower order terms are fixed by their empirical values in the mesonic sector for which evidence has been given in several places 19, 25, 26, 33. Fortunately, it turns out that the higher order stabilizers, which exclusively make up the chiral and flavor symmetric contributions to the central interaction, show little variation with respect to different baryon pairs considered. Therefore, the uncertainties associated with these terms are eliminated when we look at differences between $N N-N Y$ and $Y Y$-interactions: if some combination of higher order terms - most likely a coupling to scalar degrees of freedom - leads to a central attraction in the $N N$-case, it will also do so for the other cases.

There remain the longer range contributions from the known lower order terms: the nonlinear $\sigma$-model is responsible for the spin and flavor dependent components of the interaction, whereas the chiral and flavor symmetry breaking terms introduce further central interactions which become increasingly attractive as the strangeness contents of the participating baryons grow. The conclusion, that hyperons must be bound in deuteron like states, therefore seems

inescapable. Although our findings confirm or extend what is commonly expected, we think that we actually have added some weight to such expectations.

Since the contribution of the non-linear $\sigma$-model to the potential is also considered a reliable quantity here, we were actually in a position to predict the spins and isospins of the most attractive baryon-baryon configurations. Again, the Skyrme model result seems to agree with conclusions drawn from one boson exchange models.

\section{ACKNOWLEDGEMENTS}

We thank A. Hayashi for allowing us to upgrade his $\mathrm{SU}(2) N N$-interaction program for the present investigation and for useful discussions. One of us, B.S., wishes to thank the ITP at the University of Stellenbosch for its hospitality and a very enjoyable stay. 


\section{APPENDIX A:}

The interaction terms depend on the Euler angles of the participating baryons, with the angles in $A$ corresponding to the degrees of freedom of the first baryon, the angles in $B$ to the second. The wavefunction for an individual spin one-half baryon can be expressed as follows:

$$
\left\langle A \mid\left(\begin{array}{c}
i y \\
i_{3}
\end{array}\right)\left(\begin{array}{c}
\frac{1}{2} 1 \\
j_{3}
\end{array}\right)\right\rangle=\sum_{\{m\}} \sqrt{m} X_{\{m\}}^{i y}(-)^{\frac{1}{2}+j_{3}} D_{\left(\begin{array}{c}
i y \\
i_{3}
\end{array}\right)\left(\begin{array}{c}
\frac{1}{2} \\
-j_{3}
\end{array}\right)}^{\{m\}^{*}}(A)
$$

where the $X_{\{m\}}^{i y}$ are real amplitudes that diagonalize the rotational hamiltonian of the baryon considered 18,19, 16].

We begin with the direct matrix elements between an incoming baryon $X(A)$ interacting with a baryon $Y(B)$. The relative rotations $C=A^{\dagger} B$ contained in the $D$-functions of all flavor symmetric interaction terms translate into the following expression:

$$
\begin{aligned}
& \left\langle X^{\prime} Y^{\prime}\left|D_{\left(\begin{array}{c}
j_{1} y_{1} \\
m_{1}
\end{array}\right)\left(\begin{array}{c}
j_{2} y_{2} \\
m_{2}
\end{array}\right)}^{\{n\}}(C)\right| X Y\right\rangle= \\
& \sum_{I, y, M}\left\langle X\left|D_{\left(\begin{array}{l}
I y \\
M
\end{array}\right)\left(\begin{array}{c}
j_{1} y_{1} \\
m_{1}
\end{array}\right)}^{\{n\}}(A)\right| X^{\prime}\right\rangle^{*}\left\langle Y^{\prime}\left|D_{\left(\begin{array}{c}
I y \\
M
\end{array}\right)\left(\begin{array}{c}
j_{2} y_{2} \\
m_{2}
\end{array}\right)}^{\left\{\begin{array}{l}
n\} \\
m^{\prime}
\end{array}\right.}(B)\right| Y\right\rangle .
\end{aligned}
$$

With the conventions of de Swart [34] individual angular integrals are actually real, so we may omit the complex conjugation later. For the direct matrix elements the transferred hypercharge $y$ vanishes, and $I, j_{1}, j_{2}$ are the possible isospins and spins in the irreducible representation $\{n\}$ corresponding to hypercharge $y, y_{1}, y_{2}$ respectively. The angular integrals of the baryon-baryon interaction may all be decomposed to integrals over three $D$-functions, where isospin and spin dependence factorizes via $\mathrm{SU}(2)$ Clebsch-Gordan coefficients leaving isoscalar $\mathrm{SU}(3)$-factors [34]. We define reduced $\mathrm{SU}(3)$ matrix elements in terms of these isoscalar factors:

$$
\begin{aligned}
& \left\langle\left(i^{\prime} y^{\prime}\right)\left(\frac{1}{2} 1\right)\left\|D_{(I Y)(J 0)}^{\{n\}}\right\|(i y)\left(\frac{1}{2} 1\right)\right\rangle= \\
& \sum_{\{m\},\left\{m^{\prime}\right\}} \sqrt{\frac{m}{m^{\prime}}} X_{\{m\}}^{i y} X_{\left\{m^{\prime}\right\}}^{i^{\prime} y^{\prime}} \sum_{\gamma}\left(\begin{array}{cc|c}
\{m\} & \{n\} & \left\{m^{\prime}\right\}_{\gamma} \\
i y & I Y & i^{\prime} y^{\prime}
\end{array}\right)\left(\begin{array}{cc|c}
\{m\} & \{n\} & \left\{m^{\prime}\right\}_{\gamma} \\
\frac{1}{2} 1 & J 0 & \frac{1}{2} 1
\end{array}\right),
\end{aligned}
$$


and abbreviate the SU(2)-Clebsch-Gordan coefficients by isospin and spin operators of rank $I=1,2, J=1$. For $I=1$ we adopt the usual notation

$$
\left\langle b\left|T_{a}\right| c\right\rangle=\left(T_{a}\right)_{b c}= \begin{cases}0 & \text { for } \Lambda \\ \left(\tau_{a}\right)_{b c} & \text { for } N \text { and } \Xi \\ i \varepsilon_{a b c} & \text { for } \Sigma\end{cases}
$$

For $I=2$ it is also convenient to define an isospin quadrupole operator which will appear in the $\Sigma \Sigma$-interactions

$$
\left(\begin{array}{cc|c}
1 & 2 & 1 \\
c & a & b
\end{array}\right)=\left(Q_{a}\right)_{b c}
$$

Since all baryons considered have spin one-half, the Pauli-matrices $\sigma_{b}$ suffice to reproduce all $J=1$ matrix elements.

The different non-vanishing Euler angle matrix elements of the interaction which now can be expressed by the reduced matrix elements given in (A3) and by isoscalar factors are listed below. The spin and isospin operators embody the different possibilities for spin or isospin flips between incoming and the outgoing baryons $X^{\prime}(A)$ and $Y^{\prime}(B)$. Note that the implicit summations run over $i, j \in\{1,2,3\}$.

$$
\begin{aligned}
&\left\langle X^{\prime} Y^{\prime}\left|\left(1-\lambda D_{88}(A)\right)\left(1-\lambda^{\prime} D_{88}(B)\right)\right| X Y\right\rangle \rightarrow \\
&\left(1-\lambda\left\langle\left(i_{X} y_{X}\right)\left(\frac{1}{2} 1\right)\left\|D_{(00)(00)}^{\{8\}}\right\|\left(i_{X} y_{X}\right)\left(\frac{1}{2} 1\right)\right\rangle\right) \\
&\left(1-\lambda^{\prime}\left\langle\left(i_{Y} y_{Y}\right)\left(\frac{1}{2} 1\right)\left\|D_{(00)(00)}^{\{8\}}\right\|\left(i_{Y} y_{Y}\right)\left(\frac{1}{2} 1\right)\right\rangle\right)
\end{aligned}
$$

for arbitrary coefficients $\lambda, \lambda^{\prime}$.

$$
\begin{gathered}
\left\langle X^{\prime} Y^{\prime}\left|D_{j 8}(A) D_{j 8}(B)\right| X Y\right\rangle \rightarrow \boldsymbol{T}_{X} \cdot \boldsymbol{T}_{Y} \frac{\sqrt{\left(2 i_{X}+1\right)\left(2 i_{Y}+1\right)}}{6} \\
\left\langle\left(i_{X} y_{X}\right)\left(\frac{1}{2} 1\right)\left\|D_{(10)(00)}^{\{8\}}\right\|\left(i_{X} y_{X}\right)\left(\frac{1}{2} 1\right)\right\rangle \\
\left\langle\left(i_{Y} y_{Y}\right)\left(\frac{1}{2} 1\right)\left\|D_{(10)(00)}^{\{8\}}\right\|\left(i_{Y} y_{Y}\right)\left(\frac{1}{2} 1\right)\right\rangle \\
\left\langle X^{\prime} Y^{\prime}\left|D_{8 i}(A) D_{8 i}(B)\right| X Y\right\rangle \rightarrow \frac{1}{3} \boldsymbol{\sigma}_{X} \cdot \boldsymbol{\sigma}_{Y}\left\langle\left(i_{X} y_{X}\right)\left(\frac{1}{2} 1\right)\left\|D_{(00)(10)}^{\{8\}}\right\|\left(i_{X} y_{X}\right)\left(\frac{1}{2} 1\right)\right\rangle \\
\left\langle\left(i_{Y} y_{Y}\right)\left(\frac{1}{2} 1\right)\left\|D_{(00)(10)}^{\{8\}}\right\|\left(i_{Y} y_{Y}\right)\left(\frac{1}{2} 1\right)\right\rangle
\end{gathered}
$$




$$
\begin{array}{r}
\left\langle X^{\prime} Y^{\prime}\left|D_{j i}(A) D_{j i}(B)\right| X Y\right\rangle \rightarrow \frac{1}{3} \boldsymbol{\sigma}_{X} \cdot \boldsymbol{\sigma}_{Y} \boldsymbol{T}_{X} \cdot \boldsymbol{T}_{Y} \frac{\sqrt{\left(2 i_{X}+1\right)\left(2 i_{Y}+1\right)}}{6} \\
\left\langle\left(i_{X} y_{X}\right)\left(\frac{1}{2} 1\right)\left\|D_{(10)(10)}^{\{8\}}\right\|\left(i_{X} y_{X}\right)\left(\frac{1}{2} 1\right)\right\rangle \\
\left\langle\left(i_{Y} y_{Y}\right)\left(\frac{1}{2} 1\right)\left\|D_{(10)(10)}^{\{8\}}\right\|\left(i_{Y} y_{Y}\right)\left(\frac{1}{2} 1\right)\right\rangle
\end{array}
$$

The combination of $D$-functions where the right indices are coupled to a second rank tensor

$$
\begin{gathered}
3 D_{83}(A) D_{83}(B)-D_{8 i}(A) D_{8 i}(B) \\
3 D_{j 3}(A) D_{j 3}(B)-D_{j i}(A) D_{j i}(B)
\end{gathered}
$$

lead to the same expressions as (A8) and (A9) respectively apart from the replacement $\boldsymbol{\sigma}_{X} \cdot \boldsymbol{\sigma}_{Y} \rightarrow S_{X Y}$ where

$$
S_{X Y}=3 \boldsymbol{\sigma}_{X} \cdot \hat{R} \boldsymbol{\sigma}_{Y} \cdot \hat{R}-\boldsymbol{\sigma}_{X} \cdot \boldsymbol{\sigma}_{Y}
$$

is the tensor force operator and $\hat{R}=\mathbf{e}_{3}$ has been used.

$$
\begin{aligned}
& \left\langle X^{\prime} Y^{\prime}\left|\frac{1}{9} Q^{(I)} D_{j i}(C) D_{j i}(C)\right| X Y\right\rangle \rightarrow \frac{1}{3} \sum_{\{n\} \gamma}\left(\begin{array}{cc|c}
\{8\} & \{8\} & \{n\}_{\gamma} \\
10 & 10 & 00
\end{array}\right)^{2} q_{\{n\}}^{(I)} \times \\
& \left\{\left\langle\left(i_{X} y_{X}\right)\left(\frac{1}{2} 1\right)\left\|D_{(00)(00)}^{\{n\}}\right\|\left(i_{X} y_{X}\right)\left(\frac{1}{2} 1\right)\right\rangle\left\langle\left(i_{Y} y_{Y}\right)\left(\frac{1}{2} 1\right)\left\|D_{(00)(00)}^{\{n\}}\right\|\left(i_{Y} y_{Y}\right)\left(\frac{1}{2} 1\right)\right\rangle\right. \\
& +\boldsymbol{T}_{X} \cdot \boldsymbol{T}_{Y} \frac{\sqrt{\left(2 i_{X}+1\right)\left(2 i_{Y}+1\right)}}{6}\left\langle\left(i_{X} y_{X}\right)\left(\frac{1}{2} 1\right)\left\|D_{(10)(00)}^{\{n\}}\right\|\left(i_{X} y_{X}\right)\left(\frac{1}{2} 1\right)\right\rangle \\
& \left\langle\left(i_{Y} y_{Y}\right)\left(\frac{1}{2} 1\right)\left\|D_{(10)(00)}^{\{n\}}\right\|\left(i_{Y} y_{Y}\right)\left(\frac{1}{2} 1\right)\right\rangle \\
& \left.+\boldsymbol{Q}_{X} \cdot \boldsymbol{Q}_{Y}\left\langle\left(i_{X} y_{X}\right)\left(\frac{1}{2} 1\right)\left\|D_{(20)(00)}^{\{n\}}\right\|\left(i_{X} y_{X}\right)\left(\frac{1}{2} 1\right)\right\rangle\left\langle\left(i_{Y} y_{Y}\right)\left(\frac{1}{2} 1\right)\left\|D_{(20)(00)}^{\{n\}}\right\|\left(i_{Y} y_{Y}\right)\left(\frac{1}{2} 1\right)\right\rangle\right\} \\
& \left\langle X^{\prime} Y^{\prime}\left|\frac{1}{4} Q^{(I)}\left(D_{i i}(C) D_{j j}(C)-D_{i j}(C) D_{j i}(C)\right)\right| X Y\right\rangle \rightarrow \\
& \frac{1}{3} \boldsymbol{\sigma}_{X} \cdot \boldsymbol{\sigma}_{Y} \frac{1}{2} \sum_{\{n\} \gamma}\left(\begin{array}{cc|c}
\{8\} & \{8\} & \{n\}_{\gamma} \\
10 & 10 & 10
\end{array}\right)^{2} q_{\{n\}}^{(I)} \times \\
& \left\{\left\langle\left(i_{X} y_{X}\right)\left(\frac{1}{2} 1\right)\left\|D_{(00)(10)}^{\{n\}}\right\|\left(i_{X} y_{X}\right)\left(\frac{1}{2} 1\right)\right\rangle\left\langle\left(i_{Y} y_{Y}\right)\left(\frac{1}{2} 1\right)\left\|D_{(00)(10)}^{\{n\}}\right\|\left(i_{Y} y_{Y}\right)\left(\frac{1}{2} 1\right)\right\rangle\right. \\
& +\boldsymbol{T}_{X} \cdot \boldsymbol{T}_{Y} \frac{\sqrt{\left(2 i_{X}+1\right)\left(2 i_{Y}+1\right)}}{6}\left\langle\left(i_{X} y_{X}\right)\left(\frac{1}{2} 1\right)\left\|D_{(10)(10)}^{\{n\}}\right\|\left(i_{X} y_{X}\right)\left(\frac{1}{2} 1\right)\right\rangle \\
& \left\langle\left(i_{Y} y_{Y}\right)\left(\frac{1}{2} 1\right)\left\|D_{(10)(10)}^{\{n\}}\right\|\left(i_{Y} y_{Y}\right)\left(\frac{1}{2} 1\right)\right\rangle \\
& \left.+\boldsymbol{Q}_{X} \cdot \boldsymbol{Q}_{Y}\left\langle\left(i_{X} y_{X}\right)\left(\frac{1}{2} 1\right)\left\|D_{(20)(10)}^{\{n\}}\right\|\left(i_{X} y_{X}\right)\left(\frac{1}{2} 1\right)\right\rangle\left\langle\left(i_{Y} y_{Y}\right)\left(\frac{1}{2} 1\right)\left\|D_{(20)(00)}^{\{n\}}\right\|\left(i_{Y} y_{Y}\right)\left(\frac{1}{2} 1\right)\right\rangle\right\}
\end{aligned}
$$


As has been explained in section 3. the evaluation of antisymmetric fourth order terms from $\mathcal{L}^{(4 A)}$ is greatly simplified by the use of the operator $Q^{(I)}$, Eq. (8), for which we now give the explicit eigenvalues:

$$
q_{\{n\}}^{(I)}=\left\{\begin{array}{cc}
1 & (I)=(\widetilde{4 A}),(6) \\
3-\frac{1}{6}\left(p^{2}+q^{2}+3 p+3 q+p q\right) & (I)=(4 A)
\end{array},\right.
$$

$p$ and $q$ are related to the dimension of the representation $\{n\}$ via $n=\frac{1}{2}(p+1)(q+1)(p+q+2)$. The combination of $D$-functions where the spin indices are coupled to a second rank tensor

$$
\frac{1}{4}\left(6 D_{11}(C) D_{22}(C)-6 D_{12}(C) D_{21}(C)-D_{i i}(C) D_{j j}(C)+D_{i j}(C) D_{j i}(C)\right)
$$

leads to the same expressions as (A12) apart from the replacement $\boldsymbol{\sigma}_{X} \cdot \boldsymbol{\sigma}_{Y} \rightarrow S_{X Y}$.

For the sake of brevity, and because no other interactions will be considered, we give the exchange matrix elements only for the case where one of the interacting baryons is a nucleon $N$ the other then is a hyperon $Y$ the hypercharge of which we also designate by $Y$. For $C=A^{\dagger} B$ we make use of the relation

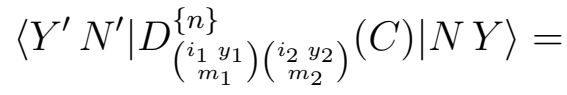

$$
\begin{aligned}
& \sum_{I, y, M}\left\langle N\left|D_{\left(\begin{array}{l}
I y \\
M
\end{array}\right)\left(\begin{array}{c}
i_{1} y_{1} \\
m_{1}
\end{array}\right)}^{\{n\}}(A)\right| Y^{\prime}\right\rangle^{*}\left\langle N^{\prime}\left|D_{\left(\begin{array}{c}
I \\
M
\end{array}\right)\left(\begin{array}{c}
i_{2} y_{2} \\
m_{2}
\end{array}\right)}^{\{n\}}(B)\right| Y\right\rangle .
\end{aligned}
$$

Again, with the conventions of de Swart [34 individual angular integrals are real, so we may omit the complex conjugation. Non-vanishing matrix elements must now transfer hypercharge $y=1-Y$ and this also restricts the possible isospins $I$ occurring in the sum of (A14).

We can give a compact representation of the non-vanishing exchange matrix elements once the different isospin cases occurring are abbreviated by 


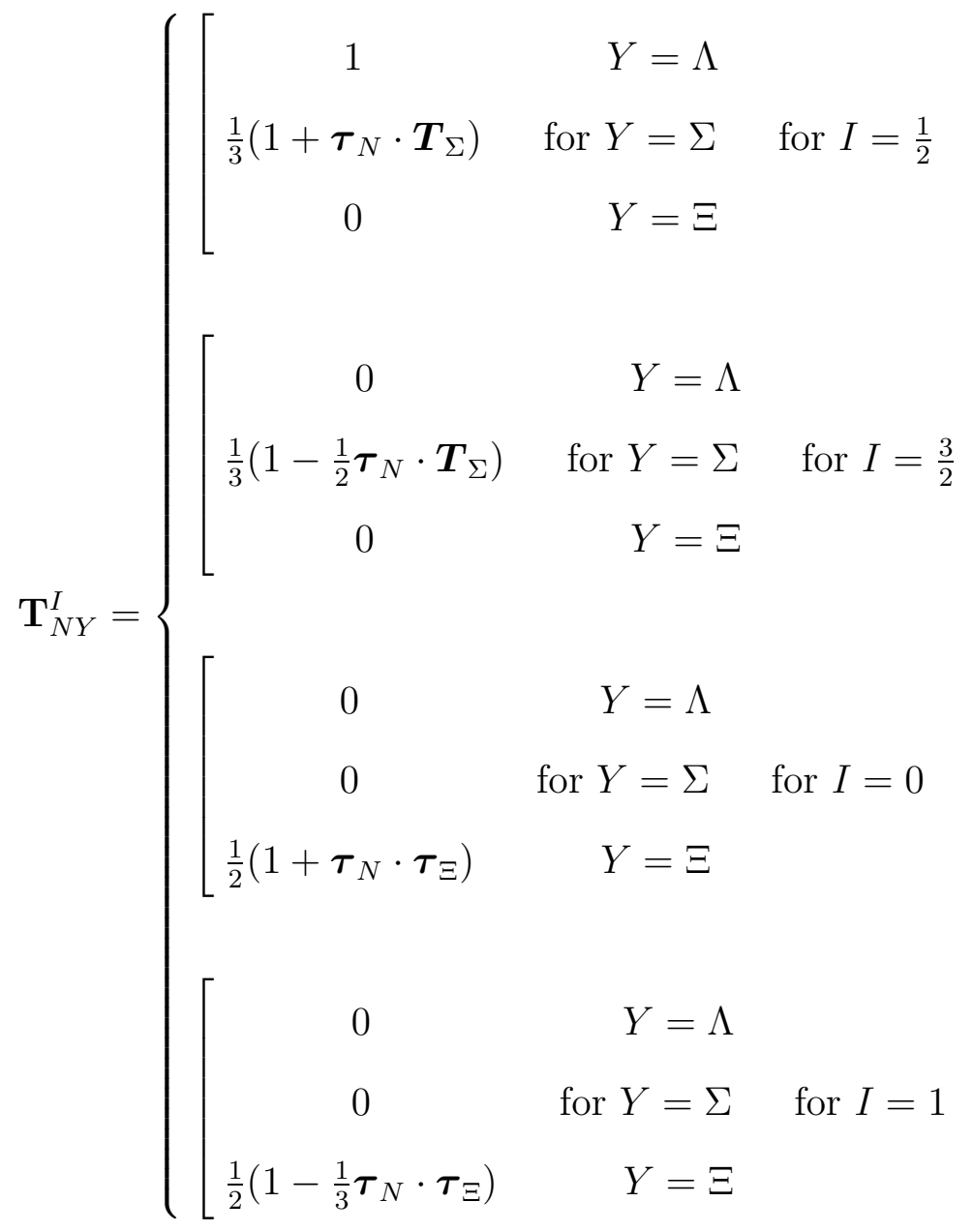

As usual, we follow our conventions for implicit sums: $i, j \in\{1,2,3\}$ and $k \in\{4,5,6,7\}$.

$$
\left\langle Y^{\prime} N^{\prime}\left|D_{i i}(C)\right| N Y\right\rangle \rightarrow \frac{1}{2}\left(1-\frac{1}{3} \boldsymbol{\sigma}_{N} \cdot \boldsymbol{\sigma}_{Y}\right) \mathbf{T}_{N Y}^{I}\left\langle\left(\frac{1}{2} 1\right)\left(\frac{1}{2} 1\right)\left\|D_{(I y)(10)}^{\{8\}}\right\|\left(i_{Y} y_{Y}\right)\left(\frac{1}{2} 1\right)\right\rangle^{2}
$$

The combination of $D$-functions where the spin indices are coupled to a second rank tensor

$$
3 D_{33}(C)-D_{i i}(C)
$$

leads to the same expression as (A15) apart from the replacement $\frac{1}{2}\left(1-\frac{1}{3} \boldsymbol{\sigma}_{N} \cdot \boldsymbol{\sigma}_{Y}\right) \rightarrow \frac{1}{3} S_{N Y}$.

$$
\begin{aligned}
& \left\langle Y^{\prime} N^{\prime}\left|D_{88}(C)\right| N Y\right\rangle \rightarrow \frac{1}{2}\left(1+\boldsymbol{\sigma}_{N} \cdot \boldsymbol{\sigma}_{Y}\right) \mathbf{T}_{N Y}^{I}\left\langle\left(\frac{1}{2} 1\right)\left(\frac{1}{2} 1\right)\left\|D_{(I y)(00)}^{\{8\}}\right\|\left(i_{Y} y_{Y}\right)\left(\frac{1}{2} 1\right)\right\rangle^{2} \\
& \left\langle Y^{\prime} N^{\prime}\left|\frac{1}{9} Q^{(I)} D_{i j}(C) D_{i j}(C)\right| N Y\right\rangle \rightarrow \\
& \frac{1}{6}\left(1+\boldsymbol{\sigma}_{N} \cdot \boldsymbol{\sigma}_{Y}\right) \sum_{\{n\} \gamma}\left(\begin{array}{cc|c}
\{8\} & \{8\} & \{n\}_{\gamma} \\
10 & 10 & 00
\end{array}\right) q_{\{n\}}^{(I)} \sum_{I}\left\langle\left(\frac{1}{2} 1\right)\left(\frac{1}{2} 1\right)\left\|D_{(I y)(00)}^{\{8\}}\right\|\left(i_{Y} y_{Y}\right)\left(\frac{1}{2} 1\right)\right\rangle^{2} \mathbf{T}_{N Y}^{I}
\end{aligned}
$$




$$
\begin{aligned}
& \left\langle Y^{\prime} N^{\prime}\left|\frac{1}{4} Q^{(I)}\left(D_{i i}(C) D_{j j}(C)-D_{i j}(C) D_{j i}(C)\right)\right| N Y\right\rangle \rightarrow \\
& \quad \frac{1}{4}\left(1-\frac{1}{3} \boldsymbol{\sigma}_{N} \cdot \boldsymbol{\sigma}_{Y}\right) \sum_{\{n\} \gamma}\left(\begin{array}{cc|c}
\{8\} & \{8\} & \{n\}_{\gamma} \\
10 & 10 & 10
\end{array}\right)^{2} q_{\{n\}}^{(I)} \sum_{I}\left\langle\left(\frac{1}{2} 1\right)\left(\frac{1}{2} 1\right)\left\|D_{(I y)(10)}^{\{8\}}\right\|\left(i_{Y} y_{Y}\right)\left(\frac{1}{2} 1\right)\right\rangle^{2} \mathbf{T}_{N Y}^{I}
\end{aligned}
$$

The combination of $D$-functions where the spin indices are coupled to a second rank tensor

$$
\frac{1}{4}\left(6 D_{11}(C) D_{22}(C)-6 D_{12}(C) D_{21}(C)-D_{i i}(C) D_{j j}(C)+D_{i j}(C) D_{j i}(C)\right)
$$

leads to the same expression as (A18) apart from the replacement $\frac{1}{4}\left(1-\frac{1}{3} \boldsymbol{\sigma}_{N} \cdot \boldsymbol{\sigma}_{Y}\right) \rightarrow \frac{1}{6} S_{N Y}$.

The flavor symmetry breaking terms finally introduce other new structures which may, however, be related to existing ones:

$$
\begin{gathered}
\left\langle Y^{\prime} N^{\prime}\left|D_{k 8}(A) D_{k 8}(B)\right| N Y\right\rangle=\left\langle Y^{\prime} N^{\prime}\left|D_{88}(C)\right| N Y\right\rangle \\
\left\langle Y^{\prime} N^{\prime}\left|D_{k i}(A) D_{k i}(B)\right| N Y\right\rangle=\left\langle Y^{\prime} N^{\prime}\left|D_{i i}(C)\right| N Y\right\rangle \\
\left\langle Y^{\prime} N^{\prime}\left|3 D_{k 3}(A) D_{k 3}(B)-D_{k i}(A) D_{k i}(B)\right| N Y\right\rangle=\left\langle Y^{\prime} N^{\prime}\left|3 D_{33}(C)-D_{i i}(C)\right| N Y\right\rangle .
\end{gathered}
$$

\section{APPENDIX B:}

In this appendix we list all interaction components of octet baryon interactions for the lagrangian given by Eqs. (1-5,20). Only the cumbersome but numerically small contributions from the term proportional to $f_{K}^{2}-f_{\pi}^{2}$ have been dropped. The parameters are those fitting the $B=0$ sector, $f_{\pi}=93.0 \mathrm{MeV}, m_{\pi}=138.0 \mathrm{MeV}, f_{K}=113.5 \mathrm{MeV}, m_{K}=495.0 \mathrm{MeV}$, with the higher order terms, $e=4.12, \chi=0, \kappa=0, \epsilon_{6}=0$, adjusted to the $N \Delta$-split in the $B=1$-sector. The Euler angle wave functions are obtained by diagonalizing the rotational and flavor symmetry breaking terms in the rigid rotator approximation.

The tables list the different radial functions subscripted by the commonly used mnemonic notation indicating the two-body spin or isospin operator combination according to (A4, A5, A10) which must be multiplied. In order to maintain uniformity of the tables there often are 
superfluous columns in the tables, e.g. the isoquadrupole-isoquadrupole interactions $U_{Q Q}$, $U_{\sigma \sigma Q Q}, U_{S Q Q}$ which can only be non-zero for the $\Sigma \Sigma$-system. For the $\Sigma \Sigma$-system they then turn out to be numerically small. 


\section{TABLES}

TABLE I.

\begin{tabular}{|c|c|c|c|c|c|c|c|c|c|}
\hline \multicolumn{10}{|c|}{ NN INTERACTION } \\
\hline$\underline{\mathrm{R}[\mathrm{fm}]}$ & $U_{0}$ & $U_{\sigma \sigma}$ & $U_{\tau \tau}$ & $U_{\sigma \sigma \tau \tau}$ & $U_{S}$ & $U_{S \tau \tau}$ & $U_{Q Q}$ & $U_{\sigma \sigma Q Q}$ & $U_{S Q Q}$ \\
\hline 0.10 & 722.1 & 0.3 & 0.3 & 15.9 & 0.0 & 0.2 & 0.0 & 0.0 & 0.0 \\
\hline 0.20 & 680.9 & 0.3 & 0.3 & 15.2 & 0.0 & 0.6 & 0.0 & 0.0 & 0.0 \\
\hline 0.30 & 618.1 & 0.3 & 0.2 & 14.1 & 0.0 & 1.2 & 0.0 & 0.0 & 0.0 \\
\hline 0.40 & 540.9 & 0.3 & 0.2 & 12.8 & 0.0 & 1.8 & 0.0 & 0.0 & 0.0 \\
\hline 0.50 & 457.0 & 0.2 & 0.2 & 11.3 & 0.0 & 2.5 & 0.0 & 0.0 & 0.0 \\
\hline 0.60 & 373.2 & 0.2 & 0.1 & 9.8 & 0.0 & 3.0 & 0.0 & 0.0 & 0.0 \\
\hline 0.70 & 295.1 & 0.2 & 0.1 & 8.3 & 0.0 & 3.3 & 0.0 & 0.0 & 0.0 \\
\hline 0.80 & 226.3 & 0.1 & 0.1 & 6.9 & 0.0 & 3.5 & 0.0 & 0.0 & 0.0 \\
\hline 0.90 & 168.5 & 0.1 & 0.1 & 5.5 & 0.0 & 3.5 & 0.0 & 0.0 & 0.0 \\
\hline 1.00 & 122.2 & 0.1 & 0.0 & 4.4 & 0.0 & 3.4 & 0.0 & 0.0 & 0.0 \\
\hline 1.10 & 86.6 & 0.1 & 0.0 & 3.4 & 0.0 & 3.1 & 0.0 & 0.0 & 0.0 \\
\hline 1.20 & 60.1 & 0.1 & 0.0 & 2.6 & 0.0 & 2.8 & 0.0 & 0.0 & 0.0 \\
\hline 1.30 & 41.1 & 0.1 & 0.0 & 2.0 & 0.0 & 2.5 & 0.0 & 0.0 & 0.0 \\
\hline 1.40 & 27.8 & 0.0 & 0.0 & 1.5 & 0.0 & 2.2 & 0.0 & 0.0 & 0.0 \\
\hline 1.50 & 18.6 & 0.0 & 0.0 & 1.1 & 0.0 & 1.9 & 0.0 & 0.0 & 0.0 \\
\hline 1.60 & 12.5 & 0.0 & 0.0 & 0.9 & 0.0 & 1.7 & 0.0 & 0.0 & 0.0 \\
\hline 1.70 & 8.3 & 0.0 & 0.0 & 0.7 & 0.0 & 1.4 & 0.0 & 0.0 & 0.0 \\
\hline 1.80 & 5.6 & 0.0 & 0.0 & 0.5 & 0.0 & 1.2 & 0.0 & 0.0 & 0.0 \\
\hline 1.90 & 3.8 & 0.0 & 0.0 & 0.4 & 0.0 & 1.1 & 0.0 & 0.0 & 0.0 \\
\hline 2.00 & 2.6 & 0.0 & 0.0 & 0.3 & 0.0 & 0.9 & 0.0 & 0.0 & 0.0 \\
\hline 2.10 & 1.7 & 0.0 & 0.0 & 0.3 & 0.0 & 0.8 & 0.0 & 0.0 & 0.0 \\
\hline 2.20 & 1.2 & 0.0 & 0.0 & 0.2 & 0.0 & 0.7 & 0.0 & 0.0 & 0.0 \\
\hline 2.30 & 0.8 & 0.0 & 0.0 & 0.2 & 0.0 & 0.6 & 0.0 & 0.0 & 0.0 \\
\hline 2.40 & 0.6 & 0.0 & 0.0 & 0.2 & 0.0 & 0.5 & 0.0 & 0.0 & 0.0 \\
\hline 2.50 & 0.4 & 0.0 & 0.0 & 0.1 & 0.0 & 0.4 & 0.0 & 0.0 & 0.0 \\
\hline 3.00 & 0.1 & 0.0 & 0.0 & 0.1 & 0.0 & 0.2 & 0.0 & 0.0 & 0.0 \\
\hline 3.50 & 0.0 & 0.0 & 0.0 & 0.0 & 0.0 & 0.1 & 0.0 & 0.0 & 0.0 \\
\hline
\end{tabular}


TABLE II.

\begin{tabular}{|c|c|c|c|c|c|c|c|c|c|}
\hline \multicolumn{10}{|c|}{ 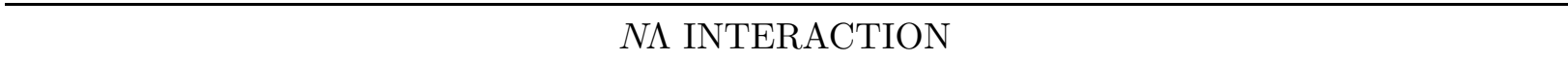 } \\
\hline $\mathrm{R}[\mathrm{fm}]$ & $U_{0}$ & $U_{\sigma \sigma}$ & $U_{\tau \tau}$ & $U_{\sigma \sigma \tau \tau}$ & $U_{S}$ & $U_{S \tau \tau}$ & $U_{Q Q}$ & $U_{\sigma \sigma Q Q}$ & $U_{S Q Q}$ \\
\hline 0.10 & 689.8 & -7.5 & 0.0 & 0.0 & 0.0 & 0.0 & 0.0 & 0.0 & 0.0 \\
\hline 0.20 & 650.8 & -7.4 & 0.0 & 0.0 & -0.1 & 0.0 & 0.0 & 0.0 & 0.0 \\
\hline 0.30 & 591.2 & -7.3 & 0.0 & 0.0 & -0.3 & 0.0 & 0.0 & 0.0 & 0.0 \\
\hline 0.40 & 518.0 & -7.1 & 0.0 & 0.0 & -0.6 & 0.0 & 0.0 & 0.0 & 0.0 \\
\hline 0.50 & 438.4 & -6.8 & 0.0 & 0.0 & -0.9 & 0.0 & 0.0 & 0.0 & 0.0 \\
\hline 0.60 & 358.9 & -6.4 & 0.0 & 0.0 & -1.2 & 0.0 & 0.0 & 0.0 & 0.0 \\
\hline 0.70 & 284.7 & -5.9 & 0.0 & 0.0 & -1.6 & 0.0 & 0.0 & 0.0 & 0.0 \\
\hline 0.80 & 219.1 & -5.4 & 0.0 & 0.0 & -1.9 & 0.0 & 0.0 & 0.0 & 0.0 \\
\hline 0.90 & 164.0 & -4.9 & 0.0 & 0.0 & -2.2 & 0.0 & 0.0 & 0.0 & 0.0 \\
\hline 1.00 & 119.6 & -4.3 & 0.0 & 0.0 & -2.5 & 0.0 & 0.0 & 0.0 & 0.0 \\
\hline 1.10 & 85.4 & -3.8 & 0.0 & 0.0 & -2.7 & 0.0 & 0.0 & 0.0 & 0.0 \\
\hline 1.20 & 59.8 & -3.2 & 0.0 & 0.0 & -2.8 & 0.0 & 0.0 & 0.0 & 0.0 \\
\hline 1.30 & 41.4 & -2.7 & 0.0 & 0.0 & -2.9 & 0.0 & 0.0 & 0.0 & 0.0 \\
\hline 1.40 & 28.4 & -2.3 & 0.0 & 0.0 & -2.9 & 0.0 & 0.0 & 0.0 & 0.0 \\
\hline 1.50 & 19.4 & -1.9 & 0.0 & 0.0 & -2.9 & 0.0 & 0.0 & 0.0 & 0.0 \\
\hline 1.60 & 13.3 & -1.6 & 0.0 & 0.0 & -2.9 & 0.0 & 0.0 & 0.0 & 0.0 \\
\hline 1.70 & 9.1 & -1.3 & 0.0 & 0.0 & -2.8 & 0.0 & 0.0 & 0.0 & 0.0 \\
\hline 1.80 & 6.3 & -1.1 & 0.0 & 0.0 & -2.7 & 0.0 & 0.0 & 0.0 & 0.0 \\
\hline 1.90 & 4.4 & -0.9 & 0.0 & 0.0 & -2.6 & 0.0 & 0.0 & 0.0 & 0.0 \\
\hline 2.00 & 3.1 & -0.7 & 0.0 & 0.0 & -2.4 & 0.0 & 0.0 & 0.0 & 0.0 \\
\hline 2.10 & 2.2 & -0.6 & 0.0 & 0.0 & -2.3 & 0.0 & 0.0 & 0.0 & 0.0 \\
\hline 2.20 & 1.6 & -0.5 & 0.0 & 0.0 & -2.2 & 0.0 & 0.0 & 0.0 & 0.0 \\
\hline 2.30 & 1.2 & -0.4 & 0.0 & 0.0 & -2.1 & 0.0 & 0.0 & 0.0 & 0.0 \\
\hline 2.40 & 0.9 & -0.3 & 0.0 & 0.0 & -1.9 & 0.0 & 0.0 & 0.0 & 0.0 \\
\hline 2.50 & 0.6 & -0.2 & 0.0 & 0.0 & -1.8 & 0.0 & 0.0 & 0.0 & 0.0 \\
\hline 3.00 & 0.1 & 0.0 & 0.0 & 0.0 & -1.3 & 0.0 & 0.0 & 0.0 & 0.0 \\
\hline 3.50 & 0.0 & 0.0 & 0.0 & 0.0 & -0.9 & 0.0 & 0.0 & 0.0 & 0.0 \\
\hline
\end{tabular}


TABLE III.

\begin{tabular}{|c|c|c|c|c|c|c|c|c|c|}
\hline \multicolumn{10}{|c|}{$N \Sigma$ INTERACTION } \\
\hline$\underline{\mathrm{R}[\mathrm{fm}]}$ & $U_{0}$ & $U_{\sigma \sigma}$ & $U_{\tau \tau}$ & $U_{\sigma \sigma \tau \tau}$ & $U_{S}$ & $U_{S \tau \tau}$ & $U_{Q Q}$ & $U_{\sigma \sigma Q Q}$ & $U_{S Q Q}$ \\
\hline 0.10 & 628.7 & 12.3 & 94.5 & 100.5 & 0.0 & 0.1 & 0.0 & 0.0 & 0.0 \\
\hline 0.20 & 592.6 & 11.5 & 89.2 & 94.9 & 0.0 & 0.5 & 0.0 & 0.0 & 0.0 \\
\hline 0.30 & 537.6 & 10.4 & 81.1 & 86.4 & 0.0 & 1.0 & 0.0 & 0.0 & 0.0 \\
\hline 0.40 & 470.0 & 9.1 & 71.1 & 76.0 & -0.1 & 1.5 & 0.0 & 0.0 & 0.0 \\
\hline 0.50 & 396.6 & 7.6 & 60.3 & 64.6 & -0.1 & 2.0 & 0.0 & 0.0 & 0.0 \\
\hline 0.60 & 323.5 & 6.2 & 49.4 & 53.3 & -0.1 & 2.4 & 0.0 & 0.0 & 0.0 \\
\hline 0.70 & 255.4 & 4.8 & 39.3 & 42.6 & -0.2 & 2.7 & 0.0 & 0.0 & 0.0 \\
\hline 0.80 & 195.4 & 3.7 & 30.3 & 33.1 & -0.2 & 2.8 & 0.0 & 0.0 & 0.0 \\
\hline 0.90 & 145.1 & 2.7 & 22.8 & 25.0 & -0.3 & 2.8 & 0.0 & 0.0 & 0.0 \\
\hline 1.00 & 104.8 & 1.9 & 16.7 & 18.4 & -0.3 & 2.7 & 0.0 & 0.0 & 0.0 \\
\hline 1.10 & 73.9 & 1.3 & 12.0 & 13.3 & -0.3 & 2.5 & 0.0 & 0.0 & 0.0 \\
\hline 1.20 & 51.0 & 0.9 & 8.4 & 9.5 & -0.4 & 2.2 & 0.0 & 0.0 & 0.0 \\
\hline 1.30 & 34.6 & 0.6 & 5.9 & 6.6 & -0.4 & 2.0 & 0.0 & 0.0 & 0.0 \\
\hline 1.40 & 23.2 & 0.4 & 4.1 & 4.6 & -0.4 & 1.7 & 0.0 & 0.0 & 0.0 \\
\hline 1.50 & 15.4 & 0.2 & 2.8 & 3.2 & -0.4 & 1.5 & 0.0 & 0.0 & 0.0 \\
\hline 1.60 & 10.2 & 0.1 & 1.9 & 2.2 & -0.4 & 1.2 & 0.0 & 0.0 & 0.0 \\
\hline 1.70 & 6.7 & 0.1 & 1.3 & 1.6 & -0.4 & 1.1 & 0.0 & 0.0 & 0.0 \\
\hline 1.80 & 4.4 & 0.0 & 0.9 & 1.1 & -0.4 & 0.9 & 0.0 & 0.0 & 0.0 \\
\hline 1.90 & 2.9 & 0.0 & 0.7 & 0.8 & -0.4 & 0.7 & 0.0 & 0.0 & 0.0 \\
\hline 2.00 & 2.0 & 0.0 & 0.5 & 0.6 & -0.3 & 0.6 & 0.0 & 0.0 & 0.0 \\
\hline 2.10 & 1.3 & 0.0 & 0.3 & 0.4 & -0.3 & 0.5 & 0.0 & 0.0 & 0.0 \\
\hline 2.20 & 0.9 & 0.0 & 0.3 & 0.3 & -0.3 & 0.4 & 0.0 & 0.0 & 0.0 \\
\hline 2.30 & 0.6 & 0.0 & 0.2 & 0.3 & -0.3 & 0.4 & 0.0 & 0.0 & 0.0 \\
\hline 2.40 & 0.4 & 0.0 & 0.1 & 0.2 & -0.3 & 0.3 & 0.0 & 0.0 & 0.0 \\
\hline 2.50 & 0.3 & 0.0 & 0.1 & 0.2 & -0.3 & 0.3 & 0.0 & 0.0 & 0.0 \\
\hline 3.00 & 0.0 & 0.0 & 0.0 & 0.1 & -0.2 & 0.1 & 0.0 & 0.0 & 0.0 \\
\hline 3.50 & 0.0 & 0.0 & 0.0 & 0.0 & -0.1 & 0.1 & 0.0 & 0.0 & 0.0 \\
\hline
\end{tabular}


TABLE IV.

\begin{tabular}{|c|c|c|c|c|c|c|c|c|c|}
\hline \multicolumn{10}{|c|}{$N \Xi$ INTERACTION } \\
\hline $\mathrm{R}[\mathrm{fm}]$ & $U_{0}$ & $U_{\sigma \sigma}$ & $U_{\tau \tau}$ & $U_{\sigma \sigma \tau \tau}$ & $U_{S}$ & $U_{S \tau \tau}$ & $U_{Q Q}$ & $U_{\sigma \sigma Q Q}$ & $U_{S Q Q}$ \\
\hline 0.10 & 552.2 & -2.9 & 2.7 & -4.1 & 0.0 & 0.0 & 0.0 & 0.0 & 0.0 \\
\hline 0.20 & 520.3 & -2.8 & 2.5 & -4.0 & 0.0 & -0.2 & 0.0 & 0.0 & 0.0 \\
\hline 0.30 & 471.7 & -2.6 & 2.3 & -3.7 & 0.0 & -0.3 & 0.0 & 0.0 & 0.0 \\
\hline 0.40 & 412.0 & -2.4 & 2.0 & -3.3 & 0.0 & -0.5 & 0.0 & 0.0 & 0.0 \\
\hline 0.50 & 347.3 & -2.1 & 1.7 & -3.0 & 0.0 & -0.7 & 0.0 & 0.0 & 0.0 \\
\hline 0.60 & 282.8 & -1.8 & 1.4 & -2.6 & 0.0 & -0.8 & 0.0 & 0.0 & 0.0 \\
\hline 0.70 & 222.8 & -1.6 & 1.1 & -2.2 & 0.0 & -0.9 & 0.0 & 0.0 & 0.0 \\
\hline 0.80 & 170.0 & -1.3 & 0.9 & -1.8 & 0.0 & -0.9 & 0.0 & 0.0 & 0.0 \\
\hline 0.90 & 125.8 & -1.1 & 0.6 & -1.5 & 0.1 & -0.9 & 0.0 & 0.0 & 0.0 \\
\hline 1.00 & 90.5 & -0.9 & 0.5 & -1.2 & 0.1 & -0.9 & 0.0 & 0.0 & 0.0 \\
\hline 1.10 & 63.5 & -0.7 & 0.3 & -0.9 & 0.1 & -0.8 & 0.0 & 0.0 & 0.0 \\
\hline 1.20 & 43.5 & -0.6 & 0.2 & -0.7 & 0.1 & -0.8 & 0.0 & 0.0 & 0.0 \\
\hline 1.30 & 29.3 & -0.5 & 0.2 & -0.5 & 0.1 & -0.7 & 0.0 & 0.0 & 0.0 \\
\hline 1.40 & 19.4 & -0.4 & 0.1 & -0.4 & 0.1 & -0.6 & 0.0 & 0.0 & 0.0 \\
\hline 1.50 & 12.7 & -0.3 & 0.1 & -0.3 & 0.2 & -0.5 & 0.0 & 0.0 & 0.0 \\
\hline 1.60 & 8.3 & -0.3 & 0.1 & -0.2 & 0.2 & -0.4 & 0.0 & 0.0 & 0.0 \\
\hline 1.70 & 5.3 & -0.2 & 0.0 & -0.2 & 0.2 & -0.4 & 0.0 & 0.0 & 0.0 \\
\hline 1.80 & 3.4 & -0.2 & 0.0 & -0.1 & 0.2 & -0.3 & 0.0 & 0.0 & 0.0 \\
\hline 1.90 & 2.2 & -0.1 & 0.0 & -0.1 & 0.2 & -0.3 & 0.0 & 0.0 & 0.0 \\
\hline 2.00 & 1.4 & -0.1 & 0.0 & -0.1 & 0.2 & -0.2 & 0.0 & 0.0 & 0.0 \\
\hline 2.10 & 0.9 & -0.1 & 0.0 & -0.1 & 0.1 & -0.2 & 0.0 & 0.0 & 0.0 \\
\hline 2.20 & 0.6 & -0.1 & 0.0 & -0.1 & 0.1 & -0.2 & 0.0 & 0.0 & 0.0 \\
\hline 2.30 & 0.4 & -0.1 & 0.0 & -0.1 & 0.1 & -0.2 & 0.0 & 0.0 & 0.0 \\
\hline 2.40 & 0.2 & 0.0 & 0.0 & 0.0 & 0.1 & -0.1 & 0.0 & 0.0 & 0.0 \\
\hline 2.50 & 0.1 & 0.0 & 0.0 & 0.0 & 0.1 & -0.1 & 0.0 & 0.0 & 0.0 \\
\hline 3.00 & 0.0 & 0.0 & 0.0 & 0.0 & 0.1 & -0.1 & 0.0 & 0.0 & 0.0 \\
\hline 3.50 & 0.0 & 0.0 & 0.0 & 0.0 & 0.1 & 0.0 & 0.0 & 0.0 & 0.0 \\
\hline
\end{tabular}


TABLE V.

\begin{tabular}{|c|c|c|c|c|c|c|c|c|c|}
\hline \multicolumn{10}{|c|}{$\Lambda \Lambda$ INTERACTION } \\
\hline$\underline{\mathrm{R}}[\mathrm{fm}]$ & $U_{0}$ & $U_{\sigma \sigma}$ & $U_{\tau \tau}$ & $U_{\sigma \sigma \tau \tau}$ & $U_{S}$ & $U_{S \tau \tau}$ & $U_{Q Q}$ & $U_{\sigma \sigma Q Q}$ & $U_{S Q Q}$ \\
\hline 0.10 & 600.8 & 12.6 & 0.0 & 0.0 & 0.0 & 0.0 & 0.0 & 0.0 & 0.0 \\
\hline 0.20 & 566.2 & 12.1 & 0.0 & 0.0 & 0.0 & 0.0 & 0.0 & 0.0 & 0.0 \\
\hline 0.30 & 513.4 & 11.3 & 0.0 & 0.0 & 0.1 & 0.0 & 0.0 & 0.0 & 0.0 \\
\hline 0.40 & 448.5 & 10.3 & 0.0 & 0.0 & 0.1 & 0.0 & 0.0 & 0.0 & 0.0 \\
\hline 0.50 & 378.1 & 9.2 & 0.0 & 0.0 & 0.1 & 0.0 & 0.0 & 0.0 & 0.0 \\
\hline 0.60 & 308.0 & 8.1 & 0.0 & 0.0 & 0.0 & 0.0 & 0.0 & 0.0 & 0.0 \\
\hline 0.70 & 242.8 & 7.0 & 0.0 & 0.0 & -0.1 & 0.0 & 0.0 & 0.0 & 0.0 \\
\hline 0.80 & 185.3 & 5.9 & 0.0 & 0.0 & -0.1 & 0.0 & 0.0 & 0.0 & 0.0 \\
\hline 0.90 & 137.3 & 4.9 & 0.0 & 0.0 & -0.2 & 0.0 & 0.0 & 0.0 & 0.0 \\
\hline 1.00 & 98.8 & 4.0 & 0.0 & 0.0 & -0.4 & 0.0 & 0.0 & 0.0 & 0.0 \\
\hline 1.10 & 69.4 & 3.3 & 0.0 & 0.0 & -0.5 & 0.0 & 0.0 & 0.0 & 0.0 \\
\hline 1.20 & 47.7 & 2.7 & 0.0 & 0.0 & -0.5 & 0.0 & 0.0 & 0.0 & 0.0 \\
\hline 1.30 & 32.1 & 2.1 & 0.0 & 0.0 & -0.6 & 0.0 & 0.0 & 0.0 & 0.0 \\
\hline 1.40 & 21.3 & 1.7 & 0.0 & 0.0 & -0.7 & 0.0 & 0.0 & 0.0 & 0.0 \\
\hline 1.50 & 14.0 & 1.4 & 0.0 & 0.0 & -0.7 & 0.0 & 0.0 & 0.0 & 0.0 \\
\hline 1.60 & 9.1 & 1.1 & 0.0 & 0.0 & -0.7 & 0.0 & 0.0 & 0.0 & 0.0 \\
\hline 1.70 & 5.9 & 0.9 & 0.0 & 0.0 & -0.7 & 0.0 & 0.0 & 0.0 & 0.0 \\
\hline 1.80 & 3.8 & 0.7 & 0.0 & 0.0 & -0.7 & 0.0 & 0.0 & 0.0 & 0.0 \\
\hline 1.90 & 2.5 & 0.6 & 0.0 & 0.0 & -0.7 & 0.0 & 0.0 & 0.0 & 0.0 \\
\hline 2.00 & 1.6 & 0.5 & 0.0 & 0.0 & -0.7 & 0.0 & 0.0 & 0.0 & 0.0 \\
\hline 2.10 & 1.0 & 0.4 & 0.0 & 0.0 & -0.7 & 0.0 & 0.0 & 0.0 & 0.0 \\
\hline 2.20 & 0.7 & 0.3 & 0.0 & 0.0 & -0.6 & 0.0 & 0.0 & 0.0 & 0.0 \\
\hline 2.30 & 0.4 & 0.2 & 0.0 & 0.0 & -0.6 & 0.0 & 0.0 & 0.0 & 0.0 \\
\hline 2.40 & 0.3 & 0.2 & 0.0 & 0.0 & -0.6 & 0.0 & 0.0 & 0.0 & 0.0 \\
\hline 2.50 & 0.2 & 0.2 & 0.0 & 0.0 & -0.5 & 0.0 & 0.0 & 0.0 & 0.0 \\
\hline 3.00 & 0.0 & 0.0 & 0.0 & 0.0 & -0.4 & 0.0 & 0.0 & 0.0 & 0.0 \\
\hline 3.50 & 0.0 & 0.0 & 0.0 & 0.0 & -0.3 & 0.0 & 0.0 & 0.0 & 0.0 \\
\hline
\end{tabular}


TABLE VI.

\begin{tabular}{|c|c|c|c|c|c|c|c|c|c|}
\hline \multicolumn{10}{|c|}{$\Sigma \Sigma$ INTERACTION } \\
\hline $\mathrm{R}[\mathrm{fm}]$ & $U_{0}$ & $U_{\sigma \sigma}$ & $U_{\tau \tau}$ & $U_{\sigma \sigma \tau \tau}$ & $U_{S}$ & $U_{S \tau \tau}$ & $U_{Q Q}$ & $U_{\sigma \sigma Q Q}$ & $U_{S Q Q}$ \\
\hline 0.10 & 555.7 & 7.0 & 23.4 & 10.0 & 0.0 & 0.1 & 0.0 & 0.0 & 0.0 \\
\hline 0.20 & 523.4 & 6.7 & 22.1 & 9.5 & 0.0 & 0.4 & 0.0 & 0.0 & 0.0 \\
\hline 0.30 & 474.2 & 6.3 & 20.1 & 8.8 & 0.0 & 0.7 & 0.0 & 0.0 & 0.0 \\
\hline 0.40 & 413.8 & 5.8 & 17.6 & 8.0 & 0.0 & 1.2 & 0.0 & 0.0 & 0.0 \\
\hline 0.50 & 348.3 & 5.2 & 14.9 & 7.1 & 0.0 & 1.6 & 0.0 & 0.0 & 0.0 \\
\hline 0.60 & 283.2 & 4.5 & 12.2 & 6.1 & 0.0 & 1.9 & 0.0 & 0.0 & 0.0 \\
\hline 0.70 & 222.6 & 3.9 & 9.7 & 5.2 & 0.0 & 2.1 & 0.0 & 0.0 & 0.0 \\
\hline 0.80 & 169.4 & 3.3 & 7.4 & 4.3 & -0.1 & 2.2 & 0.0 & 0.0 & 0.0 \\
\hline 0.90 & 124.9 & 2.7 & 5.6 & 3.5 & -0.1 & 2.2 & 0.0 & 0.0 & 0.0 \\
\hline 1.00 & 89.5 & 2.3 & 4.0 & 2.8 & -0.2 & 2.1 & 0.0 & 0.0 & 0.0 \\
\hline 1.10 & 62.4 & 1.8 & 2.9 & 2.1 & -0.3 & 2.0 & 0.0 & 0.0 & 0.0 \\
\hline 1.20 & 42.4 & 1.5 & 2.0 & 1.6 & -0.3 & 1.8 & 0.0 & 0.0 & 0.0 \\
\hline 1.30 & 28.3 & 1.2 & 1.4 & 1.2 & -0.3 & 1.6 & 0.0 & 0.0 & 0.0 \\
\hline 1.40 & 18.5 & 1.0 & 1.0 & 0.9 & -0.4 & 1.4 & 0.0 & 0.0 & 0.0 \\
\hline 1.50 & 11.9 & 0.8 & 0.6 & 0.7 & -0.4 & 1.2 & 0.0 & 0.0 & 0.0 \\
\hline 1.60 & 7.6 & 0.6 & 0.4 & 0.5 & -0.4 & 1.0 & 0.0 & 0.0 & 0.0 \\
\hline 1.70 & 4.8 & 0.5 & 0.3 & 0.4 & -0.4 & 0.9 & 0.0 & 0.0 & 0.0 \\
\hline 1.80 & 3.0 & 0.4 & 0.2 & 0.3 & -0.4 & 0.8 & 0.0 & 0.0 & 0.0 \\
\hline 1.90 & 1.8 & 0.3 & 0.1 & 0.3 & -0.4 & 0.7 & 0.0 & 0.0 & 0.0 \\
\hline 2.00 & 1.1 & 0.3 & 0.1 & 0.2 & -0.4 & 0.6 & 0.0 & 0.0 & 0.0 \\
\hline 2.10 & 0.7 & 0.2 & 0.1 & 0.2 & -0.4 & 0.5 & 0.0 & 0.0 & 0.0 \\
\hline 2.20 & 0.4 & 0.2 & 0.0 & 0.1 & -0.4 & 0.4 & 0.0 & 0.0 & 0.0 \\
\hline 2.30 & 0.2 & 0.1 & 0.0 & 0.1 & -0.3 & 0.4 & 0.0 & 0.0 & 0.0 \\
\hline 2.40 & 0.1 & 0.1 & 0.0 & 0.1 & -0.3 & 0.3 & 0.0 & 0.0 & 0.0 \\
\hline 2.50 & 0.0 & 0.1 & 0.0 & 0.1 & -0.3 & 0.3 & 0.0 & 0.0 & 0.0 \\
\hline 3.00 & 0.0 & 0.0 & 0.0 & 0.0 & -0.2 & 0.1 & 0.0 & 0.0 & 0.0 \\
\hline 3.50 & 0.0 & 0.0 & 0.0 & 0.0 & -0.2 & 0.1 & 0.0 & 0.0 & 0.0 \\
\hline
\end{tabular}


TABLE VII.

\begin{tabular}{|c|c|c|c|c|c|c|c|c|c|}
\hline \multicolumn{10}{|c|}{$\Xi \Xi$ INTERACTION } \\
\hline $\mathrm{R}[\mathrm{fm}]$ & $U_{0}$ & $U_{\sigma \sigma}$ & $U_{\tau \tau}$ & $U_{\sigma \sigma \tau \tau}$ & $U_{S}$ & $U_{S \tau \tau}$ & $U_{Q Q}$ & $U_{\sigma \sigma Q Q}$ & $U_{S Q Q}$ \\
\hline 0.10 & 508.0 & 25.2 & 23.1 & 1.1 & 0.0 & 0.0 & 0.0 & 0.0 & 0.0 \\
\hline 0.20 & 477.9 & 24.2 & 21.8 & 1.1 & 0.1 & 0.0 & 0.0 & 0.0 & 0.0 \\
\hline 0.30 & 432.2 & 22.7 & 19.8 & 1.0 & 0.1 & 0.1 & 0.0 & 0.0 & 0.0 \\
\hline 0.40 & 376.3 & 20.7 & 17.3 & 0.9 & 0.1 & 0.1 & 0.0 & 0.0 & 0.0 \\
\hline 0.50 & 315.7 & 18.5 & 14.7 & 0.8 & 0.1 & 0.2 & 0.0 & 0.0 & 0.0 \\
\hline 0.60 & 255.6 & 16.2 & 12.0 & 0.7 & 0.0 & 0.2 & 0.0 & 0.0 & 0.0 \\
\hline 0.70 & 199.8 & 14.0 & 9.5 & 0.6 & -0.1 & 0.2 & 0.0 & 0.0 & 0.0 \\
\hline 0.80 & 151.0 & 11.8 & 7.3 & 0.5 & -0.3 & 0.3 & 0.0 & 0.0 & 0.0 \\
\hline 0.90 & 110.3 & 9.9 & 5.5 & 0.4 & -0.5 & 0.3 & 0.0 & 0.0 & 0.0 \\
\hline 1.00 & 78.1 & 8.1 & 4.0 & 0.3 & -0.7 & 0.2 & 0.0 & 0.0 & 0.0 \\
\hline 1.10 & 53.6 & 6.6 & 2.8 & 0.2 & -0.9 & 0.2 & 0.0 & 0.0 & 0.0 \\
\hline 1.20 & 35.7 & 5.3 & 2.0 & 0.2 & -1.1 & 0.2 & 0.0 & 0.0 & 0.0 \\
\hline 1.30 & 23.2 & 4.3 & 1.4 & 0.1 & -1.2 & 0.2 & 0.0 & 0.0 & 0.0 \\
\hline 1.40 & 14.6 & 3.5 & 0.9 & 0.1 & -1.3 & 0.2 & 0.0 & 0.0 & 0.0 \\
\hline 1.50 & 9.0 & 2.8 & 0.6 & 0.1 & -1.4 & 0.1 & 0.0 & 0.0 & 0.0 \\
\hline 1.60 & 5.4 & 2.3 & 0.4 & 0.1 & -1.4 & 0.1 & 0.0 & 0.0 & 0.0 \\
\hline 1.70 & 3.1 & 1.8 & 0.3 & 0.0 & -1.5 & 0.1 & 0.0 & 0.0 & 0.0 \\
\hline 1.80 & 1.7 & 1.5 & 0.2 & 0.0 & -1.4 & 0.1 & 0.0 & 0.0 & 0.0 \\
\hline 1.90 & 0.9 & 1.2 & 0.1 & 0.0 & -1.4 & 0.1 & 0.0 & 0.0 & 0.0 \\
\hline 2.00 & 0.4 & 1.0 & 0.1 & 0.0 & -1.4 & 0.1 & 0.0 & 0.0 & 0.0 \\
\hline 2.10 & 0.1 & 0.8 & 0.1 & 0.0 & -1.3 & 0.1 & 0.0 & 0.0 & 0.0 \\
\hline 2.20 & -0.1 & 0.6 & 0.0 & 0.0 & -1.3 & 0.0 & 0.0 & 0.0 & 0.0 \\
\hline 2.30 & -0.2 & 0.5 & 0.0 & 0.0 & -1.2 & 0.0 & 0.0 & 0.0 & 0.0 \\
\hline 2.40 & -0.2 & 0.4 & 0.0 & 0.0 & -1.2 & 0.0 & 0.0 & 0.0 & 0.0 \\
\hline 2.50 & -0.2 & 0.3 & 0.0 & 0.0 & -1.1 & 0.0 & 0.0 & 0.0 & 0.0 \\
\hline 3.00 & -0.1 & 0.1 & 0.0 & 0.0 & -0.8 & 0.0 & 0.0 & 0.0 & 0.0 \\
\hline 3.50 & 0.0 & 0.0 & 0.0 & 0.0 & -0.6 & 0.0 & 0.0 & 0.0 & 0.0 \\
\hline
\end{tabular}




\section{REFERENCES}

[1] Th. A. Rijken, P. M. M. Maessen and J. J. de Swart, Nucl. Phys. A547, 245c (1992), and further references in this issue which covers the Shimoda Symposium on Hypernuclear and Strange Particle Physics.

[2] G. Holzwarth and B. Schwesinger, Rep. Progr. Phys. 49, 825 (1986).

[3] U.-G. Meissner and I. Zahed, in Advances in Nuclear Physics, edited by J. W. Negele and E. Vogt (Plenum Press, New York, 1986), Vol. 17, 143.

[4] V. G. Makhankov, Y. P. Rybakov and V. I. Sanyuk, The Skyrme Model - Fundamentals, Methods, Applications, Springer Series in Nuclear and Particle Physics, (SpringerVerlag, Berlin, 1993).

[5] T. S. Walhout and J. Wambach, Int. J. Mod. Phys. E 1, 665 (1992).

[6] F. G. Scholtz, B. Schwesinger and H. B. Geyer, Nucl. Phys. A561, 542 (1993).

[7] G. Kälbermann and J. M. Eisenberg, Phys. Lett. B 235, 6 (1990).

[8] G. Kälbermann and J. M. Eisenberg, Phys. Rev. D 46, 446 (1992).

[9] C. B. Dover, D. J. Milliner, A. Gal and D. H. Davis, Phys. Rev. C 44, 1905 (1991).

[10] C. B. Dover and A. Gal, in Progress in Particle and Nuclear Physics, edited by D. Wilkinson (Pergamon Press, Oxford, 1984) Vol. 12, 171-239.

[11] C. B. Dover, D. J. Milliner and A. Gal, Phys. Rep. 184, 1 (1989).

[12] D. J. Milliner, A. Gal, C. B. Dover and R. H. Dalitz, Phys. Rev. C 31, 499 (1985).

[13] R. Vinh Mau, M. Lacombe, B. Loiseau, W. N. Cottingham and P. Lisboa, Phys. Lett. B 150, 259 (1985).

[14] G. Kälbermann (private communication). 
[15] P. Jain, R. Johnson, N. W. Park, J. Schechter and H. Weigel, Phys. Rev. D 40, 855 (1989).

[16] G. Pari, B. Schwesinger and H. Walliser, Phys. Lett. B 255, 1 (1991).

[17] J. Gasser and H. Leutwyler, Nucl. Phys. B250, 465 (1985).

[18] H. Yabu and K. Ando, Nucl. Phys. B301, 601 (1988).

[19] H. Weigel, J. Schechter and N. W. Park, Phys. Rev. D 42, 3177 (1990).

[20] B. Schwesinger and H. Weigel, Phys. Lett. B 267, 438 (1991).

[21] B. Schwesinger and H. Weigel, Nucl. Phys. A450, 461, (1992).

[22] H. Yabu and K. Ando, Prog. Theor. Phys. 74, 750 (1985).

[23] A. Jackson, A. D. Jackson and V. Pasquier, Nucl. Phys. A432, 567 (1985).

[24] H. Yabu, B. Schwesinger and G. Holzwarth, Phys. Lett. B 224, 25 (1989).

[25] H. Walliser, Nucl. Phys. A524, 706 (1991).

[26] B. Schwesinger, Nucl. Phys. A537, 253 (1992).

[27] J. Schechter, Phys. Rev. D34, 868 (1986).

[28] H. Gomm, P. Jain, R. Johnson and J. Schechter, Phys. Rev. D 33, 3476 (1986).

[29] V. B. Kopeliovich, B. Schwesinger and B. Stern, Phys. Lett. B 242, 145 (1990).

[30] V. B. Kopeliovich and B. E. Stern, JETP Lett. 45, 203 (1987).

[31] J. J. M. Verbarschot, T. S. Walhout, J. Wambach and H. W. Wyld, Nucl. Phys. A461, 603 (1986).

[32] A. P. Balachandran, F. Lizzi, V. G. Rodgers and A. Stern, Nucl. Phys. B256, 525 (1985). 
[33] B. Moussalam, Ann. Phys. (N.Y.) 225, 264 (1993).

[34] J. J. de Swart, Rev. Mod. Phys. 35, 916 (1963). 


\section{FIGURES}

FIG. 1. Mass splittings for $\frac{1}{2}^{+}$and $\frac{3}{2}^{+}$baryons. Crosses indicate experimental splittings, dashed lines the spectrum calculated in the slow rotator approach, dots the results from rigidly rotated solitons. Parameters used for the rigid rotator are $f_{\pi}=93.0 \mathrm{MeV}, m_{\pi}=138.0 \mathrm{MeV}, f_{K}=113.5 \mathrm{MeV}$, $m_{K}=495.0 \mathrm{MeV}, e=4.12, \chi=0, \kappa=0, \epsilon_{6}=0$ and for the slow rotator $f_{\pi}=93.0 \mathrm{MeV}$, $m_{\pi}=138.0 \mathrm{MeV}, f_{K}=113.5 \mathrm{MeV}, m_{K}=495.0 \mathrm{MeV}, e=3.45, \chi=-0.07, \kappa=0, \epsilon_{6}=0$.

FIG. 2. Direct and exchange matrix elements for non-identical baryons.

FIG. 3. Central interaction from the flavor symmetric part of the lagrangian with fourth order stabilizing terms shown for the $N N$-system (thin full line), $\Lambda \Lambda$-system (thick full line), and $\Sigma \Sigma$ and $\Xi \Xi$-systems (dashed line). (The latter two curves practically coincide and only one is shown.) The lower quadruple of curves is obtained with the parameters $f_{\pi}=93.0 \mathrm{MeV}, m_{\pi}=138.0 \mathrm{MeV}$, $f_{K}=113.5 \mathrm{MeV}, m_{K}=495.0 \mathrm{MeV}, e=4.12, \chi=0, \kappa=0, \epsilon_{6}=0$. The upper group has $\mathcal{L}^{(4 \mathrm{~A})}$ replaced by $\mathcal{L}^{(\widetilde{4 A})}$ i.e. same parameters as before, only $\chi=1$ now.

FIG. 4. As Fig. 3, but showing the effect of alternatives to fourth order stabilizers. (As in Fig. $3 \Sigma \Sigma$ - and $\Xi \Xi$-curves practically coincide.) Upper quadruple of curves with sixth order stabilizer: $f_{\pi}=93.0 \mathrm{MeV}, m_{\pi}=138.0 \mathrm{MeV}, f_{K}=113.5 \mathrm{MeV}, m_{K}=495.0 \mathrm{MeV}, e=0, \chi=0, \kappa=0$, $\epsilon_{6}=.0125 \mathrm{MeV}^{-1}$. Lower group shows the effect of dilatons: $f_{\pi}=93.0 \mathrm{MeV}, m_{\pi}=138.0 \mathrm{MeV}$, $f_{K}=113.5 \mathrm{MeV}, m_{K}=495.0 \mathrm{MeV}, e=4.12, \chi=0, \kappa=0.03, \epsilon_{6}=0$.

FIG. 5. Central interaction from the flavor symmetry breaking part of the lagrangian for the $N N$-system (thin full line), the $\Lambda \Lambda$-system (thick full line), the $\Sigma \Sigma$-system (dotted line) and the $\Xi \Xi$-system (dashed line). The curves are obtained for the parameters $f_{\pi}=93.0 \mathrm{MeV}$, $m_{\pi}=138.0 \mathrm{MeV}, f_{K}=113.5 \mathrm{MeV}, m_{K}=495.0 \mathrm{MeV}, e=4.12, \chi=0, \kappa=0, \epsilon_{6}=0$. 
Figure 1

Mass splittings for $\frac{1}{2}^{+}$and $\frac{3}{2}^{+}$baryons. The crosses indicate the experimental splittings, the dashed lines the spectrum calculated in the slow rotator approach, dots the results from rigidly rotated solitons. For the rigid rotator we used the parameters $f_{\pi}=93.0 \mathrm{MeV}$, $m_{\pi}=138.0 \mathrm{MeV}, f_{K}=113.5 \mathrm{MeV}, m_{K}=495.0 \mathrm{MeV}, e=4.12, \chi=0, \kappa=0, \epsilon_{6}=0$ and for the slow rotator $f_{\pi}=93.0 \mathrm{MeV}, m_{\pi}=138.0 \mathrm{MeV}, f_{K}=113.5 \mathrm{MeV}, m_{K}=495.0 \mathrm{MeV}$, $e=3.45, \chi=-0.07, \kappa=0, \epsilon_{6}=0$.

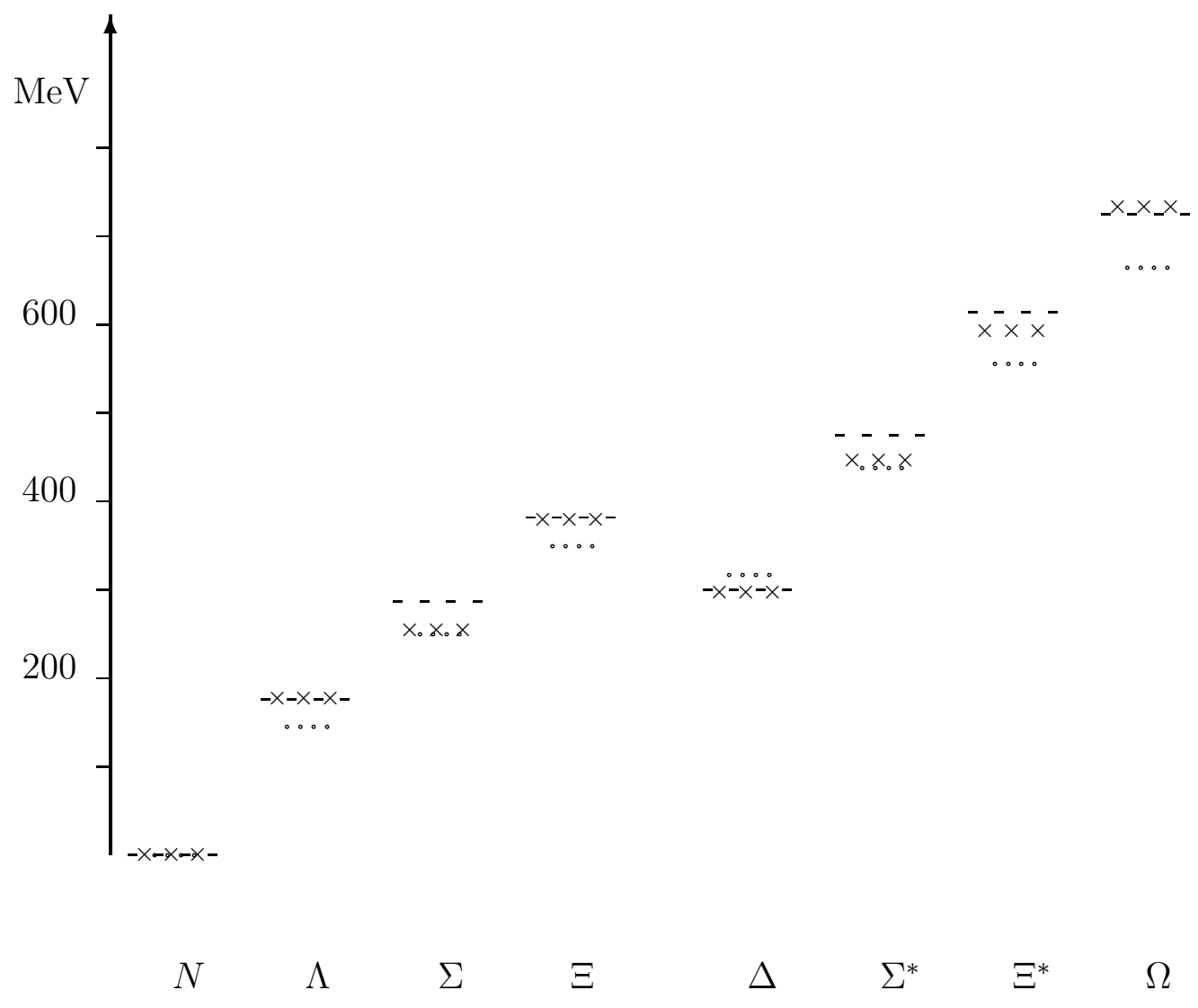


Figure 2

Direct and exchange matrix elements for non-identical baryons.
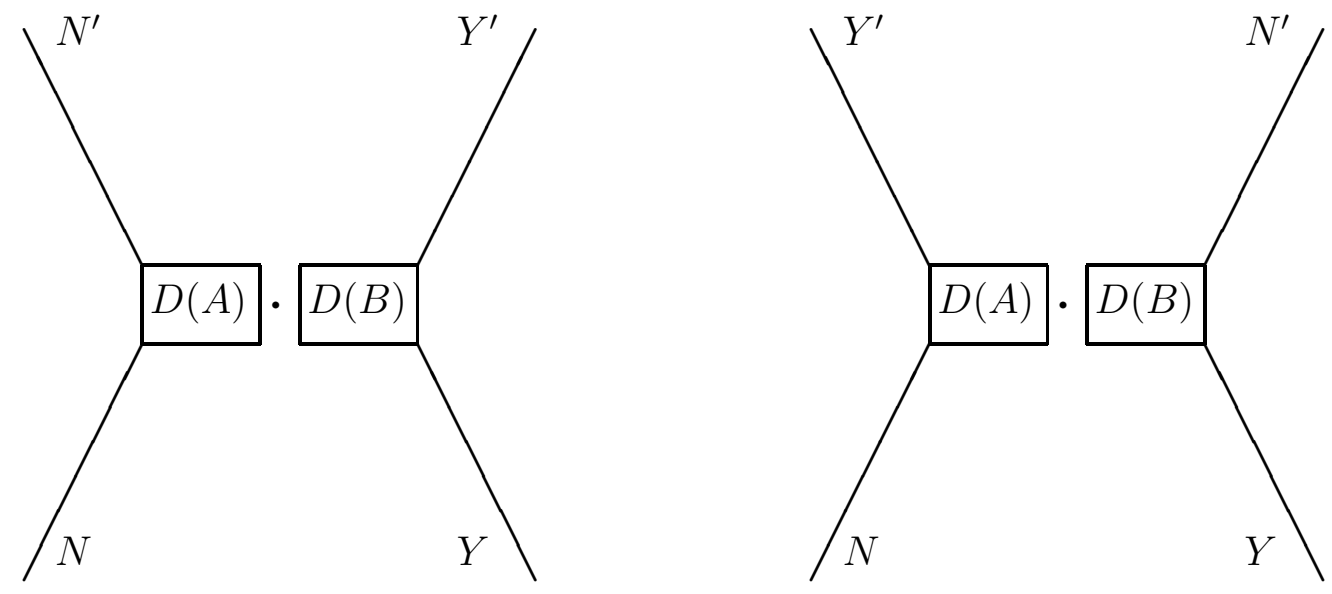
This figure "fig1-1.png" is available in "png" format from: http://arxiv.org/ps/nucl-th/9408002v1 
This figure "fig1-2.png" is available in "png" format from: http://arxiv.org/ps/nucl-th/9408002v1 
This figure "fig1-3.png" is available in "png" format from: http://arxiv.org/ps/nucl-th/9408002v1 